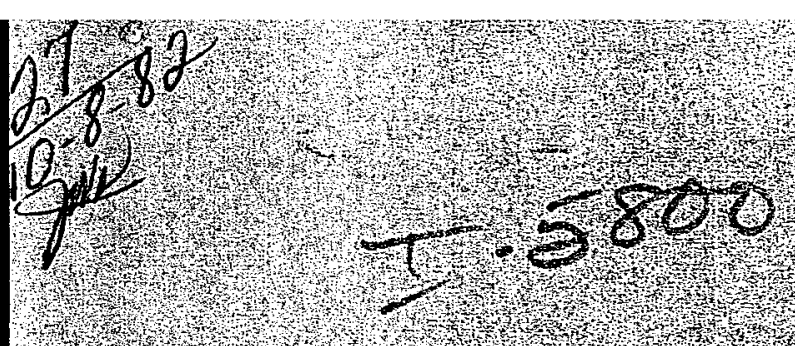

\title{
Iist of DOE Radioisotope
}

Customers with summary of

Radioiso to pe shipments,

FY 1981

compiled by

1.5. Burlison

\section{MASTER}

September 1982

Prepared for

Office of Heallotand Envitonmental Research

Office of thersy research

1.S. Departinent of thergy

Wasingtong \& 6,20545

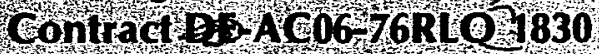

Pacific Northyestlaboratory

Operated for the US. Department of Energy

by battelle Memoral Institute 


\section{DISCLAIMER}

This report was prepared as an account of work sponsored by an agency of the United States Government. Neither the United States Government nor any agency Thereof, nor any of their employees, makes any warranty, express or implied, or assumes any legal liability or responsibility for the accuracy, completeness, or usefulness of any information, apparatus, product, or process disclosed, or represents that its use would not infringe privately owned rights. Reference herein to any specific commercial product, process, or service by trade name, trademark, manufacturer, or otherwise does not necessarily constitute or imply its endorsement, recommendation, or favoring by the United States Government or any agency thereof. The views and opinions of authors expressed herein do not necessarily state or reflect those of the United States Government or any agency thereof. 


\section{DISCLAIMER}

Portions of this document may be illegible in electronic image products. Images are produced from the best available original document. 
PNL -4177

UC-23

PNL- -4177

DE83 000303

LIST OF DOE RADIOISOTOPE CUSTOMERS WITH

SUMMARY OF RADIOISOTOPE SHIPMENTS, FY 1981

Compi led by

J. S. Burlison

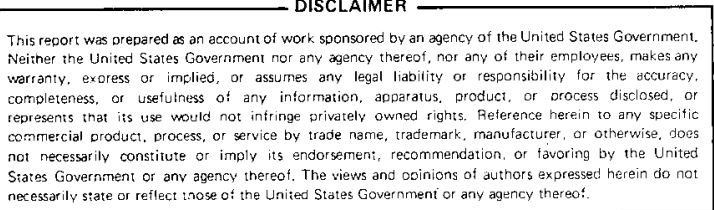

September 1982

Prepared for

Office of Health and Environmental Research

Office of Energy Research

U.S. Department of Energy

Washington, D.C. 20545

Contract DE-AC06-76RL 01830

Pacific Northwest Laboratory

Richland, Washington 99352

This document is PUBLICLY RELEASABLE 


\section{ACK NOWL EDGMENTS}

The compiler thanks the following laboratories and DOE Area Offices for their annual contributions from which this report is compiled.

Argonne National Laboratory

Brookhaven National Laboratory

Hanford Engineering Development Laboratory

Idaho Operations office/EG\&G Idaho, Inc.

Los Alamos Scientificil aboratory

Oak Ridge National Laboratory

Pacific Northwest Laboratory

Savannah River Plant /OOE

UNC Nuclear Industries, Inc.

Previous reports in this series are:

TIO-2160 (FY 1964$)$
ORNL-IIC-6 (FY 1965)
ORNL-IIC-9 (FY 1966)
ORNL-IIC-13 (FY 1967$)$
ORNL-IIC-17 (FY 1968$)$
ORNL-IIC-27 (FY 1969$)$

ORNL- IIC-32 (FY 1970)

ORNL-IIC-38 (FY 1971)

BNWL - 1774 (FY 1972)

BNWL - 1824 (FY 1973)

BNWL - 1902 (FY 1974)

BNWL-1993 (FY 1975)
BNWL -2147 (FY 1976)

PNL-2572 (FY 1977)

PNL-2930 (FY 1978)

PNL-3274 (FY 1979)

PNL-3839 (FY 1980)

Other reports covering stable isotope sales include:

"Stable Isotope Sales: Mound Facility Customer and Shipment Summary, FY 1980", MLM-2843.

"Stable Isotope Sales: Mound Facility Customer and Shipment Sumary, FY 1979", ML M-2718.

"Stable Isotope Customer List and Summary of Shipments, FY 1981", ORNL/TM-8302.

"Stable Isotope Customer List and Summary of Shipments, FY 1980," ORNL/TM-7764.

"Stable Isotope Customer List and Sumnary of Shipments, FY 1979," ORNL/TM-7214.

Similar reports covering stable isotope distribution have also been prepared and are available from the National Technical Information Center, U.S. Department of Commerce, 5285 Port Royal Rd, Springfield, VA 22161. 


\section{CONTENTS}

ACKNOWLEDGMENTS

- $\mathbf{i} \mathbf{i}$

PREVIOUS REPORTS

- i i i

LIST OF DOE RADIOISOTOPE CUSTOMERS WITH SUMMARY OF RADIOISOTOPE SHIPMENTS FY 1981

1. ISOTOPE SUPPLIER, FACILITY CONTACT AND ISOTOPES OR SERVICE SUPPLIED

3

2. ALPHABETICAL LIST OF CUSTOMERS, SUPPLIERS AND ISOTOPES PURCHASED - 5

3. ALPHABETICAL LIST OF ISOTOPES CROSS-REFERENCED TO CUSTOMER NUMBER . 23

4. GEOGRAPHIC LOCATION OF RADIOISOTOPE CUSTOMERS - $\quad 27$

5. RADIOISOTOPE SALES AND TRANSFERS - FY 1981. . 29 
The eighteenth edition of the radiosiotope customer list was prepared at the request of the Office of Health and Environmental Research, Office of Energy Research, Department of Energy (DOE). This document lists DOE's radioisotope production and distribution activities by its facilities at Argonne National Laboratory; Pacific Northwest Laboratory; Brookhaven National Laboratory; Hanford Engineering Development Laboratory; Idaho Operations Office; Los Alamos Scientific Laboratory; Oak Ridge National Laboratory; Savannah River Laboratory; and UNC Nuclear Industries, Inc.

The information is divided into five sections:

1. Isotope Suppliers, Facility, Contacts and Isotopes or Services Supplied. Section 1 lists suppliers of isotopes supplied, and the contact person for each DOE facility. If a reader needs more specific information that is not supplied in this report about isotope sales or shipments for a particular facility, he or she should contact the person listed for that facility.

2. Alphabetical List of Customers, and Isotopes Purchased. Section 2 is an alphabetical list of domestic and foreign customers and their addresses. Each customer has a customer number. This number is used to cross-reference data in the other sections of the report. Isotope purchases are listed after each customer's address, and the laboratory supplying each isotope is indicated by a letter abbreviation set off by parentheses.

3. Alphabetical List of Isotopes Cross-Referenced to Customer Numbers. Section 3 alphabetically lists isotopes and cross-references them to customer numbers. This section provides a quick idea of the number of customers purchasing a particular isotope.

4. Geographical Location of Radioisotope Customers. Section 4 is an alphabetical list of states and foreign countries that is cross-referenced to customer numbers and indicates the geographical concentration of isotope users.

5. Radioisotope Sales and Transfers-FY 1981. Section 5 summarizes the FY-1981 radioisotode shioment activities of the DOE laboratory facilities listed in Section 1. A table alphabetically lists the isotopes and their suppliers. Data on shipments, quantities, and dollar values of each isotope are listed under domestic, foreign, and project (DOE facilities) categories. 

1. ISOTOPE SUPPLIER (a) FACILITY CONTACT, AND ISOTOPES OR SERVICES SUPPLIED

The following abbreviations are used in this listing:

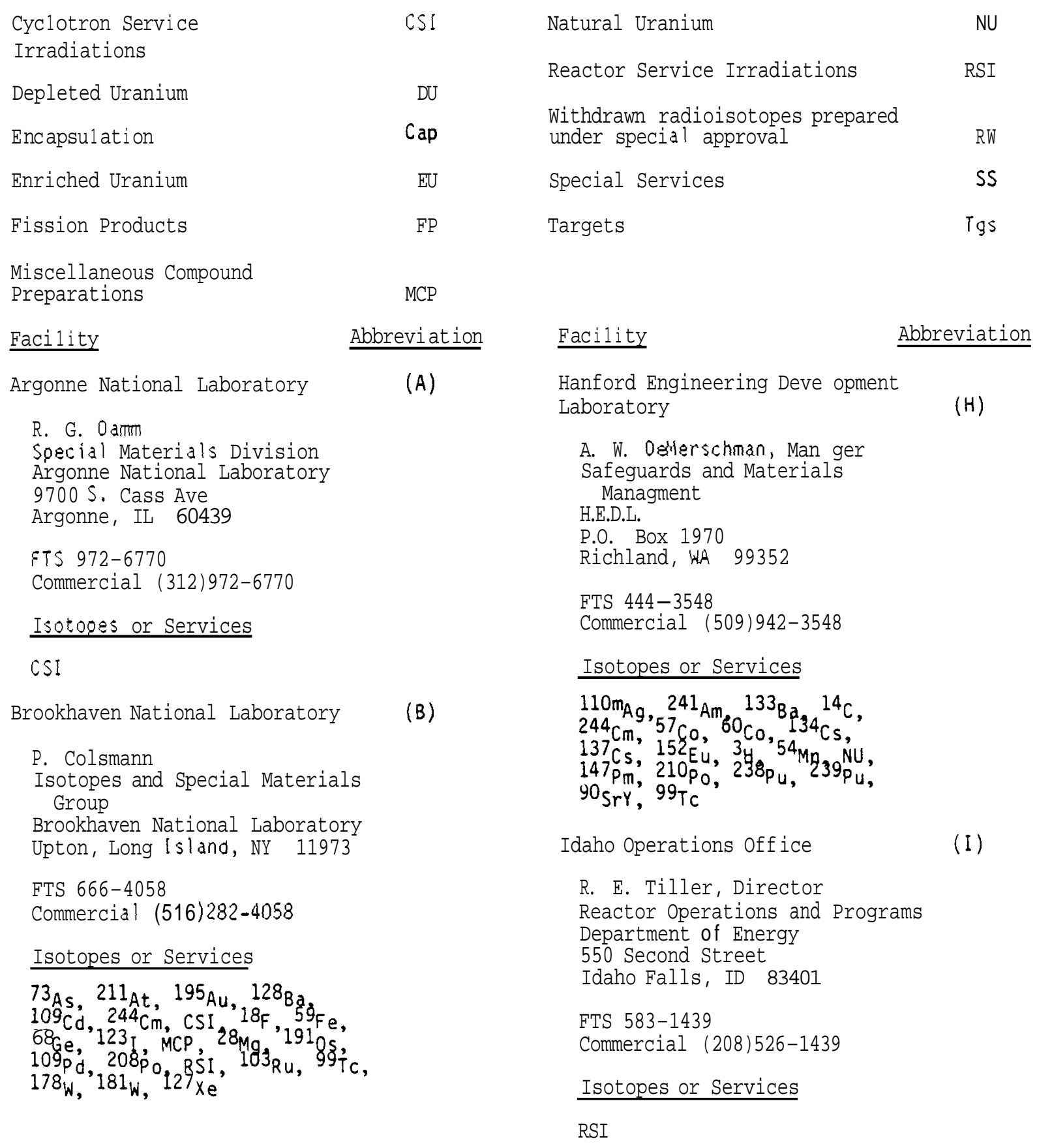

(a) All entries for Argonne, UNC, and Idaho are either reactor service irradiations, cyclotron service irradiations, or both with no processed isotopes being sold; entries for Savannah River represent material for resale and loans. 


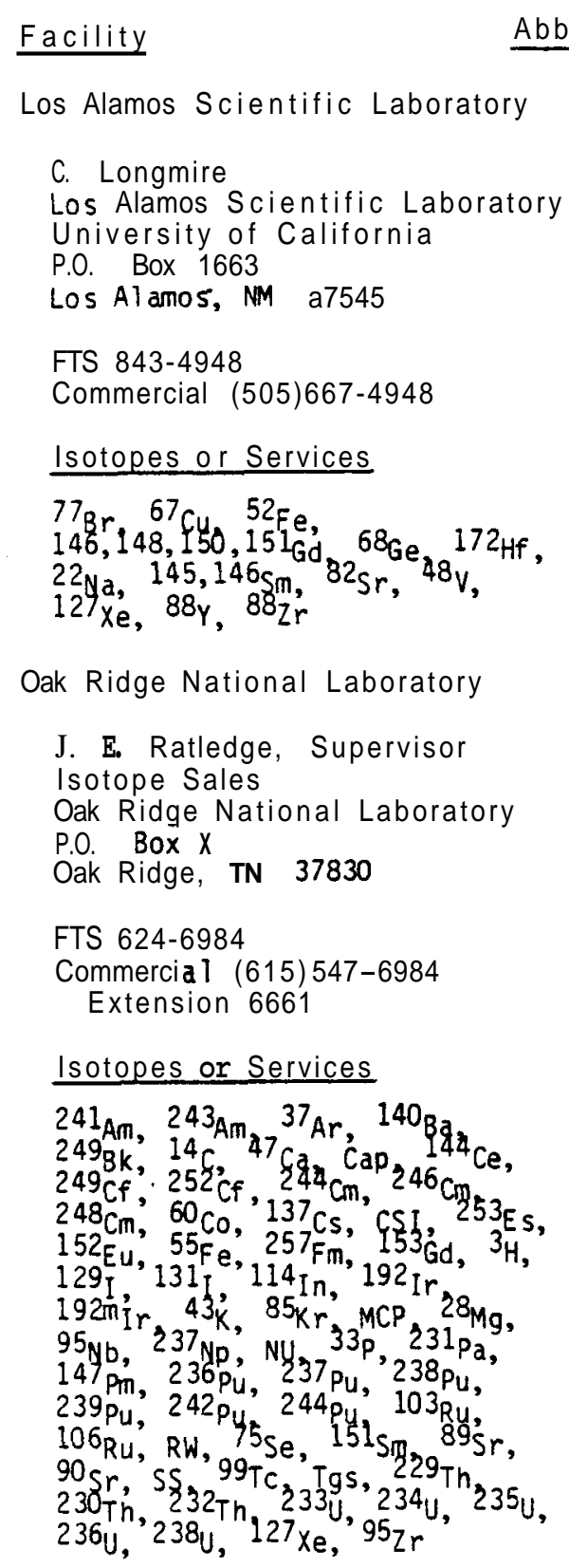

(L)

\section{ation}


2. ALPHABETICAL LIST OF CUSTOMERS, SUPPLIERS AND ISOTOPES PURCHASED

The letter following each isotope or service entry is the supplier abbreviation listed in Section 1.

DOMESTIC CUSTOMERS

1 Aerojet Nuclear Company

550 Second Street

P.O. Box 1845

Idaho Falls, Idaho 83401

$241 \mathrm{Am}(0),{ }^{243} \mathrm{Am}(0),{ }^{140} \mathrm{Ba}(0)$, ${ }^{144} \mathrm{Ce}(0),{ }^{252} \mathrm{Cf}(0),{ }^{244} \mathrm{Cm}(0)$, $60 \mathrm{Co}(0), 153_{\mathrm{Gd}(0)} 192 \mathrm{Ir}(0)$, SS $(0), 237 \mathrm{Ng}(0), 236 \mathrm{pu}_{\mathrm{y}}(0)$. $239 \mathrm{Pu}(0), 242 \mathrm{Pu}(0), 103_{\mathrm{Ru}}(0)$, $230 \mathrm{Th}(0), 233_{U(0)},{ }^{95} \mathrm{Zr}(0)$

2 AIRCO Inc. Central Research Laboratories Murray Hill, New Jersey 07974

\section{$85_{k r}(0)$}

3 American Hoechst Corporation P.O. Box 2500

Sumerville, New Jersey 08876

\section{$85 \mathrm{Kr}(0)$}

4 Amersham Corporation Arlington Heights, Illinois 60005

$$
153_{\mathrm{Gd}(0)},{ }^{192} \operatorname{Ir}(0),{ }^{85} \mathrm{Kr}(0)
$$

5 Amtech Incorporated

938 W. Grant Street

Tucson, Arizona 85705

$85_{K r}(0), S \$(0)$

6 Analog Devices Semiconductor 829 Woburn Street Wi lmington, Massachusetts 01887

\section{$85_{\mathrm{Kr}}(0)$}

7 Analytics Incorporated

P.0. Box 27565

Atlanta, Georgia 30327

$$
127 \times \mathrm{e}\left(8^{*}\right)
$$

8 Argonne National Laboratory 9700 South Cass Avenue Argonne, Illinois 60439

$$
\begin{aligned}
& { }^{249} \mathrm{Bk}(0){ }_{253} \mathrm{Cf}(0) \text { } 5 \mathrm{Tgs}(0) \text {, } \\
& \text { Cap (0), } 253 E_{s}(0),{ }^{2} E_{u}(0) \text {, } \\
& { }^{59} \mathrm{Fe}(\mathrm{B}),{ }^{237} \mathrm{~Np}(0), 239 \mathrm{Pu}(0) \text {, } \\
& 106 \mathrm{Ru}(0),{ }^{230} \mathrm{Th}(0),{ }^{232} \mathrm{Th}(0) \text {, } \\
& 235_{U}(0),{ }^{238} U(0),{ }^{9} 5_{Z r}(0), \operatorname{RSI}(B)
\end{aligned}
$$

(*) Oak Ridge billed.
9 Argonne National Laboratory P.0. Box 2528 Idaho Falls, Idaho 83401

$\operatorname{Tgs}(0), 238_{U}(0)$

10 Automation Industries, Inc. P.0. Box 245

Phoenixville, Pennsylvania 19460

$$
192 \operatorname{Ir}(0)
$$

11 Battelle Pacific Northwest Laboratories P.0. Box 999

Richland, Washington 99352

$241_{\mathrm{Am}}(\mathrm{H}),{ }^{133} \mathrm{Ba}(\mathrm{H}), 244 \mathrm{Cm}(\mathrm{H})$, $244 \mathrm{Cm}(\mathrm{O}),{ }^{60} \mathrm{Co}(\mathrm{H}){ }^{134} \mathrm{Cs}(\mathrm{H})$, $137 \mathrm{Cs}(\mathrm{H}){ }^{3} \mathrm{H}(\mathrm{H}),{ }^{59} \mathrm{Mn}(\mathrm{H})$, $\mathrm{NU}(\mathrm{H}),{ }^{147} \mathrm{Pm}(\mathrm{H}),{ }^{210} \mathrm{PO}(\mathrm{H})$, $23 P_{P u}(O), 238_{P u}(H), 239_{P u}(H)$, $97 \mathrm{Ru}(U),{ }^{90} \mathrm{SrY}(\mathrm{H})$, Tgs $(0)$,

12 Bell Telephone Laboratories 600 Mountain Avenue Murray Hill, New Jersey 07974

$$
{ }^{181_{W}(B),} \quad{ }^{73} \text { As (B) }
$$

13 Boeing Company Power Generation Group 9.0. Box 3979 Seattle, Washington 98124

$236_{p_{u}(0),} 233 U(0), 235_{U(0)}$

14 Brookhaven National Laboratory Upton, Long Island, New York 11973

$147 \mathrm{Pm}(0), 8 \mathrm{~W}(0), S S(0), 241_{A m}(0)$, Cao $(0), 244 \mathrm{Cm}(0), 68 \mathrm{Ge}(\mathrm{L}), 3 \mathrm{H}(0)$,
ss $(0), \operatorname{Tgs}(0), 229 \mathrm{Th}(0), 127 \mathrm{Xe}(\dot{L})$

15 California Institute of Technology 2101 E. California Boulevard Pasadena, California 91109

${ }^{77_{B r}(L),}{ }^{235_{U}(0),}{ }^{127} \mathrm{Xe}\left(\mathrm{B}^{*}\right)$

16 California State University Department of Chemistry Fullerton, California 92624

$67_{\mathrm{Cu}}(\mathrm{L})$ 
17 California State Univers it y Northbridge, California 91330

$48 \mathrm{~V}(\mathrm{~L})$

18 Campbell Pacific Nuclear Corporation 130 So. Buchanan Circle

Pacheco, California 94553

${ }^{137} \mathrm{Cs}(\mathrm{B})$

19 Carnegie Institution of Washington Washington, D.C. 20008

${ }^{144} \mathrm{Ce}(0),{ }^{152_{E u}(0)},{ }^{151_{S m}(0)}$

20 Carnegie \& Mellon University

5000 Forbes

Pittsburgh, Pennsylvania 15213

$\operatorname{Tgs}(0),{ }^{252} \mathrm{Cf}(0)$,

21 Case Western Reserve University 2220 Circle Drive

Cleveland, Ohio 44106

$3_{H(0), 28_{M g}\left(B^{*}\right)}$

22 Chevron Research Company

Richmond, California 94802

$172_{H F(L)}, 172_{\text {Lu(L) }}$

23 Children's Hospital

Division of Nuclear Medicine

300 Longwood Road

Boston, Massachusetts 02115

$191_{0 s}(B)$

24 Children's Hospital Medical Center

Radiation Control

20 Blackf an Street

Boston, Massachusetts 02115

$127 \mathrm{Xe}\left(B^{*}\right)$

25 Colorado State University

Fort Collins, Colorado 80521

$241_{A m(0)}$

26 Columbia University

Health Physics Office

520 West 120 Street

New York, New York 10027

$\operatorname{csi}(0)$

27 Columbia University

630 West 168 Street

New York, New York 10032

${ }^{109} p_{d}(B)$
28 Conoco, Incorporated

P.O. Box 1785

Uvalde, Texas 78801

\section{${ }^{89} \mathrm{Sr}(0)$}

29 Conoco, Incorporated

Research \& Development Department

P.O. Box 1267

Ponca City, Oklahoma 74601

${ }^{85} \mathrm{Kr}(0),{ }^{103_{\mathrm{Ru}}(0),{ }^{89} \mathrm{Sr}(0)}$

30 Continental Testing Laboratories

763 U.S. Highway 17 \& 92

Fern Park, Florida 32730

$85_{\mathrm{Kr}}(0)$

31 Cornel1 University

Purchasing

120 Maple Avenue

Ithaca, New York 14850

${ }^{47} \mathrm{Ca}(0)$

32 Creighton University

2410 California

Omaha, Nebraska 68131

${ }^{47} \mathrm{Ca}(0)$

33 Cryogenic Rare Gas Laboratories, Inc. 730 South 13th Street

Newark, New Jersey 07103

$85 \mathrm{Kr}(0)$

34 Dow Chemical Company

Larkin Laboratory

$1691 \mathrm{~N}$. Swede Road

Midland, Michigan 48640

$3_{H(B)}$

35 E. I. du Font de Nemours \& Company, Inc.

Savannah River Plant

Aiken, South Carolina 29801

${ }^{252} \mathrm{Cf}(0),{ }^{248} \mathrm{Cm}(0), \mathrm{SS}(0)$

36 E. A. D. Metallurgical, Inc.

71 Pearce Avenue

Tonawanda, New York 14150

$241_{A m(0), C a p(0)}$

37 East Carolina University

Purchasing Department

Greenville, North Carolina 27834

${ }^{89} \mathrm{Sr}(0)$ 
38 Eastman Kodak Company Kodak Park Works

Rochester, New York 14650

129 I $(0)$

39 East Virginia Medical Authority

Radiation Safety

519 Front Drive

Norfolk, Virginia 23510

${ }^{22} \mathrm{Na}(\mathrm{B})$

40 EG\&G Idaho, Inc.

P.O. Box 1625

Idaho Falls, Idaho 83415

RSI ( I)

41 E. M. R. Photo Electric

Div. of Weston Instruments

P.0. Box 44

Princeton, New Jersey 08540

$3_{H}(0)$

42 Exxon Nuclear Idaho Company

P.O. Box 2800

Idaho Falls, Idaho 83401

$\operatorname{RSI}(I)$

43 Fairchild Semiconductor

P.O. Box 790

Mountain View, California 94040

$85 \mathrm{Kr}(0)$

44 Fermi National Accelerator Lab

Batavia, Illinois 60510

${ }^{60} \mathrm{Co}(\mathrm{B})$

45 Florida State University

Tallahassee, Florida 32306

$237_{\mathrm{Np}}(0)$

46 Ford Aerospace \& Communication Corp.

Division of Ford Motor Company

3939 Fabian Way

Palo Alto, California 94303

$85_{\mathrm{Kr}(0)}$

47 Gamma Industries, Inc.

P.0. Box 2543

Baton Rouge, Louisiana 70821

$192 \operatorname{Ir}(0)$
48 Gamma Industries

9320 Travenor

P.0. Box 34526

Houston, Texas 77034

$241_{A m}(0), C a p(0)$

49 General Electric Company

Lunchburg, Virginia 24502

$85_{\mathrm{Kr}}(0)$

50 General Electric Company

P.O. Box 254

San Jose, California 95103

${ }^{144} \mathrm{Ce}(0),{ }^{137} \mathrm{Cs}(0),{ }^{153} \mathrm{Gd}(0)$, $\operatorname{MCP}(0), S S(0), 232$ in $(0)$

51 General Electric Company

Vallecitos Nuclear Company

Vallecitos Road

Pleasanton, California 94566

$\operatorname{RSI}(I),{ }^{252} \mathrm{Cf}(\mathrm{S})$

52 General Electric Company

General Electric Drive

Waynesboro, Virginia 22980

$85 \mathrm{Kr}(0)$

53 General Electric Research \& Development

Bldg. KI

Schenectady, New York 12301

$\mathrm{RSI}(\mathrm{B})$

54 General Nucleonics Division, TYCO Laboratories

2811 Metropol itan Place

Pomona, California 91767

$85_{k r}(0)$

55 General Semiconductor, Inc. 2001 West 10th Place

Temple, Arizona 85281

$85_{\mathrm{Kr}}(0)$

56 George Washington University 23 \& I Street N.W.

Washington, D.C. 20037

$77_{\mathrm{Br}}(\mathrm{L}),{ }^{123} \mathrm{I}(\mathrm{B})$

57 Georgetown University 3800 Reservoir Road, N.W. Washington, D.C. 20007

${ }^{89} \operatorname{sr}(0)$ 
58 Georgia Institute of Technology

Nuclear Research Center

900 Atlantic Drive N.W.

Atlanta, Georgia 30332

$127 \times \mathrm{e}\left(B^{\star}\right)$

59 Gollob Analytical Service Corporation 47 Industrial Road

Berkeley Heights, New Jersey 07922

${ }^{3}(0),{ }^{85} \mathrm{Kr}(0)$

60 Gulf Energy and Environmental Systems P.0. Box 608

San Diego, California 92112

${ }^{89} \mathrm{Sr}(0), \mathrm{Ss}(0),{ }^{95} \mathrm{Zr}(0)$

61 Gulf Nuclear Incorporated

202 Medical Center

vebster, Texas 77598

${ }^{241} 1_{A m}(0), C_{a p}(0),{ }^{137} \mathrm{Cs}(0)$,

$153 \mathrm{Gd}(0)$, $192 \mathrm{Ir}(0)$

62 Harvard Medical School

Shields Warren Laboratory

Boston, Massachusetts 02115

$211_{A t(B)}, 18_{F(B)}$

63 Hughes Ai rcraft Company

P.O. Box 92907

Los Angeles, California 90009

${ }^{85} \mathrm{Kr}(0)$

64 I C I America, Inc.

Atlas Aerospace Division

Valley Forge, Pennsylvania 19481

$85_{K r}(0)$

65 I C N \& N B Co.

26201 Miles Road

Cleveland, Ohio 44128

${ }^{137} \mathrm{Cs}(0), \operatorname{CSI}(0){ }_{153} \mathrm{Gd}(0)$, $3 \mathrm{H}(0), 33 \mathrm{P}(0), 147 \mathrm{p}_{\mathrm{g}}(0)$, ${ }^{108_{R u}(0),}{ }^{75} \mathrm{Se}(0),{ }^{89} \mathrm{Sr}(0)$, $90 \mathrm{Sr}(0)$

66 Indiana University

Bloomington, Indiana 47401

${ }^{14} \mathrm{C}(0), \mathrm{sS}(0)$

67 Indiana University

630 W. New York Street

Indianapolis, Indiana 46202

${ }^{47} \mathrm{Ca}(0)$
68 Industrial Nuclear Company

1124 Chess Drive

Forest City, California 94404

${ }^{192} \operatorname{Ir}(0)$

69 INTEL Corporation

3065 Bowers Avenue

Santa Clara, California 95051

$85_{\mathrm{Kr}}(0)$

70 International Antex, Inc.

2630 California Street

Mountain View, California 94040

$\operatorname{MCP}(0),{ }^{147} P_{m}(0)$

71 Iowa State University

Ames, Iowa 50010

$129 I(0), \operatorname{MCP}(0), 234 U(0)$,

$235 \cup(0), 236 U(0), 238 U(0)$,

$192 \operatorname{Ir}(\mathrm{B}),{ }^{182} \mathrm{Ta}(\mathrm{B})$

72 Isomedix Inc.

P.O. Box 177

25 Eastmans Road

Parsippany, New Jersey 07054

${ }^{137} \operatorname{Cs}(0), \operatorname{MCP}(0)$

73 Isotope Products Laboratories

404 South Luke Street

Burbank, California 91502

${ }^{243} \mathrm{Am}(0),{ }^{144} \mathrm{Ce}(0),{ }^{252} \mathrm{Cf}(0)$, ${ }^{137} \mathrm{Cs}(0), \mathrm{CSI}(0),{ }^{55} \mathrm{Fe}(0)$, $129 \mathrm{I}(0), 85 \mathrm{Kr}(0), 231 \mathrm{~Pa}(0)$ 208 Po(B) 236pu(0), $239 \mathrm{pu}(0)$, ${ }^{106 \mathrm{Ru}(0)}{ }^{89} \mathrm{Sr}(0),{ }^{90} \mathrm{Sr}(0)$, Igs(0), $230 \mathrm{Th}(0), 235 \mathrm{y}(0)$,

74 Isotec, Incorporated

7542 McE wem Road

Centerville, Ohio 45459

$\operatorname{MCP}(0)$

75 Isovac Engineering Company 6220 San Fernando Road Glendale, California 92104

$85 \mathrm{Kr}(0)$

76 Jewish Hosptial of $5 t$. Louis 216 S. Kingshighway Boulevard st. Louis, Missouri 63110

${ }^{47} \mathrm{Ca}(0)$ 
77 John Hopkins University

Baltimore, Maryland 21218

$\operatorname{MCP}(0)$

78 John Hopkins Medical Institute

Division of Nuclear Medicine

$622 \mathrm{~N}$. Washington Street

Baltimore, Maryland 21205

$77 \operatorname{Br}(\mathrm{L}),{ }^{123} \mathrm{I}(\mathrm{B})$

79 Kaman Nuclear

1700 Garden of the Gods Roads

Colorado Springs, Colorado 80907

$3_{H}(0)$

80 KMS Fusion, Inc.

3941 S. Research Park Drive

P.0. Box 1567

Ann Arbor, Michigan 48106

$3_{H}(0)$

81 Land Seas Corporation

1212 Avenue of the Americas

New York, New York 10036

${ }^{129} \mathrm{I}(0)$

82 Lee Environmental Lab

2030 Wright Avenue

Richmond, California 94304

$3_{H(B)}$

83 Los Angeles County USC Med. Center 1200 N. State Street

Box 112 G.H.

Los Angeles, California 90033

${ }^{47} \mathrm{Ca}(0)$

84 Lovelace Clinic

4800 Gibson Boulevard, S.E.

Albuquerque, New Mexico 87108

${ }^{243} \mathrm{Cm}(0), \mathrm{SS}(0)$

85 Loyola University Medical Center 2160 So. First Avenue

Maywood, Illinois 60153

$\operatorname{CSI}(\mathrm{A})$

86 Mallinckrodt Chemical Works

3600 N. Second Street

St. Louis, Missouri 63160

$\operatorname{CSI}(0),{ }^{75} \operatorname{Se}(0), \operatorname{ss}(0), 127 \mathrm{Xe}\left(B^{*}\right)$
87 Mallinckrodt Nuclear

P.0. Box 5840

St. Louis, Missouri 63134

$127 \mathrm{Xe}\left(B^{*}\right)$

88 Marubeni America Corporation

14 Henderson Orive

West Caldwell, New Jersey 07006

$242 p u(0)$

89 Massachusetts General Hospital

Boston Massachusetts 02114

${ }^{47} \mathrm{Ca}(0),{ }^{18} \mathrm{~F}(\mathrm{~B}),{ }^{68_{\mathrm{Ge}}(\mathrm{L})}$

90 Massachusetts Institute of Technology Box 69

Cambridge, Massachusetts 02139

${ }^{47} \mathrm{Ca}(0),{ }^{3} \mathrm{H}(0)$

91 Mayo Foundation

Rochester, Minnesota 55901

${ }^{47} \mathrm{Ca}(0)$

92 Measurex Corporation

10475 Imperial Avenue

Cupertino, California 95014

$85_{K r}(0)$

93 Medical College of Wisconsin 8700 W. Wisconsin Avenue

Milwaukee, Wisconsin 53226

$43_{K}(0)$

$94 \quad$ Medilab

312 N.E. 15th Street

Miami, Florida 33132

$235 u(0)$

95 Merck and Company

Rahway, New Jersey 07065

${ }^{60} \mathrm{Co}(0)$

96 Methodist Hospital

1604 W. Capitol

Indianapolis, Indiana 46202

$127 \mathrm{xe}\left(B^{*}\right)$

97 Michael \& Reese Hospital 29th Street and Ellis Avenue Chicago, Illinois 60616

${ }^{47} \mathrm{Ca}(0)$ 
98 Minnesota Mining Manufacturing Co.

P.O. Box 3121

St. Paul, Minnesota 53101

${ }^{137} \mathrm{Cs}(0),{ }^{85} \mathrm{Kr}(0), \operatorname{MCP}(0)$,

$95 \mathrm{Nb}(0)$

99 Mitsus and Company

c/o USA, Inc.

200 Park Avenue

New York, New York 10017

$234 \mathrm{U}(0)$

100 Monolithic Memories Incorporated

1165 East Argues Avenue

Sunnyvale, California 94086

$85_{k r}(0)$

101 Monsanto Research Corporation

Dayton Laboratory

151 Nicholas Road

Dayton, Ohio 45407

$241_{\text {Am }}(0), C a p(0)$,
$252_{\text {Cf }(S)}{ }^{238} 8_{P u(0)}$,

102 Moravek Biochemicals Inc.

577 Mercury Lane

Brea, California 92621

$3_{H}(0)$

103 Motorola Inc.

5005 East McDowell Road

Phoenix, Arizona 85008

$85 \mathrm{Kr}(0)$

104 Mount Sinai Hospital

Fifth Avenue and 100th Street

New York, New York 10029

$\operatorname{MCP}(0),{ }^{82} \mathrm{Sr}(\mathrm{L})$

105 National Semiconductor

1850 West Grant Road

Suite 114

Tucson, Arizona 85705

$85_{\mathrm{Kr}(0)}$

106 National Semiconductor Corporation 2900 Semiconductor Drive

Santa Clara, California 95051

$85_{\mathrm{Kr}}(0)$

106a Neutron Products, Inc.

P.0. Box 68

Bickerson, Maryland 20573

${ }^{60} \mathrm{Co}(0)$
107 New England Medical Center Boston, Massachusetts 02111

$77 \mathrm{Br}(\mathrm{L})$

108 New England Nuclear Corporation 575 Albany Street

Boston, Massachusetts 02118

$241_{A m}(0),{ }^{14} C(0), C a p(0)$,

${ }^{144} \mathrm{Ce}(0), 137 \mathrm{Cs}(0), \operatorname{CSI}(0)$,

$55 \mathrm{Fe}(0), 153 \mathrm{Gd}(0), 3 \mathrm{H}(0)$,

$129 \mathrm{I}(0), 85_{\mathrm{Kr}}(0), 33 \mathrm{p}(0)$,

$75 \mathrm{Se}(0), 151 \mathrm{Sm}(0), \mathrm{Ss}(0)$,

${ }^{99} \mathrm{Tc}(0),{ }^{95} \mathrm{Zr}(0)$

109 New England Nuclear Corporation Radiopharmaceutical Div.

601 Treble Cove Road

Billerica, Massachusetts 01862

$195_{\mathrm{Au}}(\mathrm{B}), 128_{\mathrm{Ba}}(\mathrm{B}),{ }^{109} \mathrm{Cd}(\mathrm{B})$, $58 \mathrm{Co}(\mathrm{B}), 68 \mathrm{Ge}\left(\mathrm{B}^{\star}\right),{ }^{\prime} 68_{\mathrm{Ge}}(\mathrm{L})$, $22_{\mathrm{Na}(L)}, 178_{\mathrm{W}}(\mathrm{B}),{ }^{2} 88_{Y}(\mathrm{~L})$

110 New York Hospital

Nuclear Medicine

525 East 68th Street

New York, New York 10021

$\operatorname{RSI}(B)$

111 New York University Medical Center

550 First Avenue

New York, New York 10016

${ }^{237} \mathrm{~Np}(0), \operatorname{Tgs}(0)$

112 New York University Medical Center

A. J. Lanza Lab

Long Meadow Road

Tuxedo, Texas 10987

$\operatorname{RSI}(B)$

113 North Carolina Baptist Hospital

Nuclear Medicine

300 S. Hawthorne

Winston-Salem, North Carolina 27103

${ }^{127} \mathrm{Xe}\left(\mathrm{B}^{*}\right)$

114 North Carolina Memorial Hospital

NCNB Plaza, 6th Floor

Chapel Hill, North Carolina 27514

${ }^{127} \mathrm{Xe}\left(\mathrm{B}^{*}\right)$

115 Northeastern Ohio Universities

College of Medicine

4209 State Route 44

Rootstown, Ohio 44272

$28 \mathrm{Mg}\left(\mathrm{B}^{*}\right)$ 
116 Nuclear Radiation Development, Inc. 2937 A I t Blvd Grand Island, New York 14072

$241_{A m(0), C a p}(0), 3_{H}(0)$

117 Nuclear Research Corporation 2 Richwood Place

Oenville, New Jersey 07834

${ }^{239} P_{u}(0), \operatorname{Tgs}(0)$

118 Nuclear Sources and Services

P.0. Box 14023

Houston, Texas 77021

$241_{A m(0),} \operatorname{Cap}(0)$

119 Nuclepore Corporation

7035 Commerce Circle

Pleasanton, California 94566

$\operatorname{Tgs}(0), 235 u(0)$

120 Oak Ridge Associated Universities

P.O. Box 117

Oak Ridge, Tennessee 37830

$243_{A m}(0), C a p(0), \operatorname{CSI}(0)$

121 Ohio State University

Research Foundation

1314 Kinnear Road

Columbus, Ohio 43212

${ }^{14} \mathrm{C}(0),{ }^{89} \mathrm{Sr}(0)$

122 Ohio University

Athens, Ohio 45701

$3_{H}(0)$

123 Oregon State University

Corvallis, Oregon 97330

$236_{U(0)}$

124 Potter and Brumfield

Princeton, Indiana 47570

$85 \mathrm{kr}(0)$

125 Princeton University

Plasma Physics Laboratory

Accounts Payable Department

P.O. Box 451

Princeton, New Jersey 08544

${ }^{252} \mathrm{Cf}(0),{ }^{85} \mathrm{Kr}(0), \mathrm{SS}(0)$
126 Princeton University James Forrestal Campus Princeton, New Jersey 08544

${ }^{137} \mathrm{Cs}(\mathrm{B}),{ }^{24} \mathrm{I}_{\mathrm{Am}(0)}, \operatorname{Tgs}(0)$

127 Proctor and Gamble Company

P.O. Box 175

Groesbecic Station

Cincinnati, Ohio 45239

$85 \mathrm{Kr}(0)$

128 Purdue University

Pharmacy Annex

Lafayette, Indiana 47907

${ }^{47} \mathrm{Ca}(0),{ }^{99} \mathrm{Tc}(0)$

129 Radiation Management Corporation

Suite 30,

3508 Market Street

Philadelphia, Pennsylvania 19104

$85_{k r}(0)$

130 Raytheon Company

350 Ellis Street

Mountain View, California 94042

$85 \mathrm{kr}(0)$

131 RCA

Raritan, New Jersey 08869

$85_{K r(0)}$

132 Reactor Experiments, Inc.

963 Terminal Way

San Carlos, California 94070

$237 \mathrm{~Np}(0), \operatorname{Tgs}(0),{ }^{235_{U}(0)}$, $238 \mathrm{U}(0)$

133 Rensselaer Polytechic Institute

1108 th Street

Troy, New York 12181

$28_{\mathrm{Mg}}\left(\mathrm{B}^{*}\right), \mathrm{RSI}(\mathrm{B})$

134 Reuter \& Stokes Electronics Components 18530 South Miles Parkway

Warrensville Heights

Cleveland, Ohio 44128

$234 \cup(0)$

135 Reynolds Electric Engineering Company P.O. Box 14400

Las Vegas, Nevada 89114

${ }^{243} \mathrm{Am}(0),{ }^{244} \mathrm{Cm}(0)$ 
136 Rockwell International

Atomics Internationa 1 Di vi sion

Rocky Flats Plant

P.0. Box 464

Golden, Colorado 80401

$242 p_{u}(0), 233 v(0)$

137 Rockwell Hanford Operations

Richland, Washington 99352

${ }^{90} \mathrm{SrY}(\mathrm{H}),{ }_{137} \mathrm{Cs}(\mathrm{H}), 110 \mathrm{~m}_{\mathrm{Ag}}(\mathrm{H})$,

99 TC(H), $152_{\mathrm{Eu}(\mathrm{H})}, 106_{\mathrm{Ru}(0)}$

138 Rush Presbyterian - St. Luke's

Medical Center

Nuclear Medicine

1753 W. Congress Parkway

Chicago, Illin ois 60612

${ }^{52} \mathrm{Fe}$ (B)

139 Rutgers State University

Busch Campus

P.0. Box 939

Piscataway, New Jersey 08854

RS I ( B )

140 Safety Light Corporation 4150 \& A Old Berwick Road

Bloomsburg, Pennsyl vani a 17815

$3_{H}(0)$

141 Sandia Corporation

P.0. Box 5800

Albuquerque, Noxico 87115

$85 \mathrm{Kr}(0)$

142 Schwarz Bio \& Research, Inc.

Mount ain View Avenue

Orangeburg, New York 10962

${ }^{3} \mathrm{H}(0)$

143 Science Applications

3 Choke Cherry Road

Rockvi 1le, Maryland 20850

${ }^{237} \mathrm{~Np}(0), \operatorname{Tgs}(0)$

144 Science Applications

1200 Prospect Street

La Jolla, California 92037

$237_{\mathrm{Np}}(0), \operatorname{Tgs}(0)$

145 Self \& Powered Lighting, Ltd.

8 Weschester Plaza

Elmsford, Nork York 10523

${ }^{3} \mathrm{H}(0)$
146 She 11 Development Company

1410 45th Street

Eneryvi 1le, Calif orni a 94608

$85 \mathrm{kr}(0)$

147 J. L. Shepherd and Associates

1614 Victory Boulevard

Glendale, California 91201

${ }^{137} \operatorname{Cs}(0), \operatorname{MCP}(0)$

148 Signetics Corporation

1275 South 800 East

Orem, Utah 84057

$85_{k r}(0)$

149 Signetics Incorporated

811 East Argues Avenue

Sunnyvale, California 94086

$85 x r(0)$

150 Silicon General Incorporated

11651 Monarch Street

Garden Grove, California 92641

$85_{\mathrm{Kr}}(0)$

151 Siliconix Incorporated

2201 Laurelwood Road

Santa Clara, California 95054

$85_{\mathrm{kr}}(0)$

152 Sloan Ketterng Cancer Center

Central Isotope Lab

1275 York Ave.

Now York, New York 10032

${ }^{103} \mathrm{Ru}(8),{ }^{82} \mathrm{Sr}(\mathrm{L})$

153 Source Production and Equipment Co., Inc.

625 0x ley Street

Kenner, Loui si ana 70062

${ }^{192} \operatorname{Ir}(0)$

154 Southern Californi a University of Uni versity Park

Los Angeles, California 90007

$\operatorname{MCP}(0)$

155 Sprague Electric Company

North Adams, Massachusetts 01247

$85_{\mathrm{k} r}(0)$ 
156 E. R. Squibb and Sons

P.O. Box 70

East Brunswick, New Jersey 08816

${ }^{75} \mathrm{Se}(0),{ }^{99} \mathrm{Tc}_{\mathrm{c}}(0)$

157 E. R. Squibb and Sons

New Brunswick, New Jersey 08903

${ }^{82} \mathrm{Sr}(\mathrm{L})$

158 State University of New York 3435 Main Street

Buffalo, New York 14214

${ }^{89} \mathrm{Sr}(0),{ }^{99} \mathrm{Tc}(0)$

159 State University of New York at Stony Brook

Department of Earth Sciences

Stony Brook, New York 11794

\section{RS I ()}

160 State University of New York at Stony Brook

University Hospital

Stony Brook, New York 11794

\section{$127_{\mathrm{Xe}}\left(\mathrm{B}^{*}\right)$}

161 Statek Corporation

1200 Alvaret Avenue

Orange, California 92668

$85 \mathrm{Kr}(0)$

Technica 1 Operations

South Avenue

Burlington, Massachusetts 01803

${ }^{192} \operatorname{Ir}(0), \mathrm{SS}(0)$

163 Teledyne Isotopes

Nuclear Systems Division

110 W. Timonium Road

Timonium, Maryland 21093

$\operatorname{MCP}(0),{ }^{90} \mathrm{Sr}(0)$

164 Teledyne Isotopes.

123 Woodland Avenue

Westwood, New Jersey 07675

${ }^{89} \mathrm{Sr}(0)$

165 Texas A \&

College of Agriculture

Box 156

Kingsville, Texas 78363

${ }^{147} \mathrm{Ca}(0)$
166 Texas A \& M University

P.0. Box 44

College Station, Texas 77843

$\mathrm{NU}(0)$

167 Texas Instruments, Inc.

Science Services Division

P.O. Box 5012, Mail Stop 6

Dallas, Texas 75222

${ }^{3} \mathrm{H}(0), 85_{\mathrm{Kr}}(0)$

168 Trio \& Tech International

2040 W. Lincoln Street

Burbank, California 91504

$85_{\mathrm{Kr}}(0)$

169 UNC Nuclear Industries

P.0. Box 490

Richland, Washington 99352

${ }^{60} \mathrm{Co}(\mathrm{H}),{ }^{57} \mathrm{Cg}(\mathrm{H}),{ }^{110 \mathrm{~m}_{\mathrm{Ag}}(\mathrm{H}),}$

170 Union Carbide Corporation Nuclear Division

Oak Ridge Gaseous Diffusion Plant

P.0. Box $P$

Oak Ridge, Tennessee 37830

$85 \mathrm{kr}(0)$

171 Union Carbide Corporation Nuclear Division

Oak Ridge National Laboratory

P.0. Box $X$

Oak Ridge, Tennessee 37830

${ }^{241_{A m}(0)} 241_{A m}(S),{ }^{47} \mathrm{Ca}(0)$,

$24 \mathrm{Cm}^{\mathrm{B}}(\mathrm{B}){ }^{244} \mathrm{C} \mathrm{Cm}(\mathrm{O}),{ }^{253} \mathrm{Cs}(\mathrm{O})$,

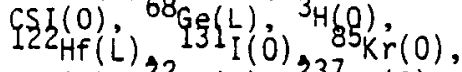

$\operatorname{MCP}(0),{ }^{22} \mathrm{Na}(\mathrm{L}),{ }^{237} \mathrm{~Np}(0), \mathrm{NU}(0)$,

239 Pu(0), 242 Pu(0), RU(0),

${ }^{75} \mathrm{Se}(0),{ }^{89} \mathrm{Sr}(0), \quad s \mathrm{~s}(0)$,

23) $c(0)$, Tgs $(0), 232 \mathrm{Th}(0), \mathrm{Th}(\mathrm{P})$,

172 Union Carbide Corporation

Nuclear Division

$Y$ \& 12 Plant

P.0. Box $Y$

Oak Ridge, Tennessee 37830

CSI $(0), 153_{\mathrm{Gd}}(0), 114 \operatorname{In}(0)$,

$192 \operatorname{Ir}(0), \operatorname{MCP}(0), 147 \mathrm{Pm}(0), \mathrm{SS}(0)$ 
173 Union Carbide Corporation

Nuclear Division

Paducah Gaseous Diffusion Plant

Paducah, Kentucky 42002

$233 u(0), 236 u(0)$

174 Union Carbide Corporation Sterling Forest Research Center P.O. Box 324

Tuxedo, New York 10987

$75 \mathrm{Se}(0)$

175 Unit Process Assemblies, Inc.

$53 \& 15$ 37th Avenue

Woodside, New York 11377

${ }^{147} \mathrm{Pm}(0),{ }^{90} \mathrm{Sr}(0)$

176 United States Air Force

McClellan Air Force Base, California 95652

$37_{\mathrm{Ar}(0)}$

177 United States Department of

Agriculture

Research Service

P.O. Box 53326

New Orleans, Louisianna 70150

$241_{A m(0)}$

178 United State Department of Energy 376 Hudson Street Fifth Floor

New York, New York 10014

$236 \mathrm{pu}(0)$

179 United States Department of Energy Chicago Operations Office

Finance Division

9800 South Cass Avenue

Argonne, IIIinois 60439

$243_{\mathrm{Am}(0)}$

180 United States Department of Energy New Brunswick Laboratory

9800 South Cass Avenue

Argonne, Illinois 60439

$P u(P), \quad$ OU $(P)$

181 United States Department of Energy Richland Operations Office

P.0. Box 550

Richland, Washington 99352

Cap (0), 236pu(0), 238pu(0)
United States Department of Energy

Denver Federal Center

Denver, Colorado 80225

$\mathrm{Pu}(\mathrm{P})$

183

United States Geological Survey

Bldg. 21

Denver, Colorado 80225

${ }^{237} \mathrm{~Np}(0),{ }^{239} \mathrm{Pu}(0)$

184 United States Geological Survey

12201 Sunrise Valley Drive

Reston, Virginia 22092

${ }^{252} \mathrm{Cf}(\mathrm{S})$

185 United State Food and Drug Administration

12 th and C Streets, S.W.

Washington, D.C. 20204

${ }^{47} \mathrm{Ca}(0),{ }^{99} \mathrm{Tc}(0),{ }^{99} \mathrm{Tc}(\mathrm{B})$

186 United States National Aeronautics and Space Administration

John F. Kennedy Space Center, Florida 32899

\section{$238 \mathrm{pu}(\mathrm{s})$}

187

United States National Aeronautics and Space Administration

Goddard Space Flight Center

Glendale Road

Greenbelt, Maryland 20771

$85_{\mathrm{Kr}}(0)$

188 United States National Aeronautics and Space Administration

Manned Spacecraft Center

Houston, Texas 77058

${ }^{47} \mathrm{Ca}(0), \operatorname{RSI}(\mathrm{B})$

189

United States National Bureau of Standards

Connecticut Avenue and Van Ness St. N.W.

Washington, D.C. 20234

${ }^{252} \mathrm{Cf}(0),{ }^{3} \mathrm{H}(0){ }^{129} \mathrm{I}(0)$,

\$s(0), $237_{\mathrm{Np}}(0), 236 \mathrm{Pu}(0)$,

$99 \mathrm{Tc}(0), \operatorname{Tgs}(0), \mathrm{EU}(\mathrm{P})$

190 United States National Bureau of Standards

Rec. Room, Bldg. 301

Route 270 \& Quince Orchard Road

Gaithersburg, Maryland 20760

$127 \mathrm{Xe}\left(B^{*}\right)$ 
191 United States National Institute of Health

Neurosurgery Branch

Bldg. 10, Room 11 N242

Bethesda, Maryland 20014

$18 \mathrm{~F}(\mathrm{~B})$

192 United States National Institute of Health

Bldg. 21

Bethesda, Maryland 20014

$68_{G e}\left(B^{*}\right),{ }^{82} S r(L),{ }^{127} X e\left(B^{*}\right)$,

193 United States Navy

Charleston Naval Shipyard

United States Naval Base

Charleston, South Carolina

$127 \mathrm{Xe}\left(\mathrm{B}^{*}\right)$

194 United States Navy

National Naval Medical Center

Bethesda, Maryland 20014

$127 \times e\left(B^{*}\right)$

195 United States Navy

Naval A ir Propulsion Center

1440 Parkway Avenue

Trenton, New Jersey 08628

$85 \mathrm{Kr}(0)$

196 United States Navy

Naval Regional Medical Center

8750 Mountain Boulevard and

Building 505

Oakland, California 94627

$127 \times e\left(B^{*}\right)$

197 United States Navy

Naval Research Laboratory

Supply Officer

Washington, D.C. 20375

\section{${ }^{49} V(B)$}

198 United States Navy

Naval Weapons Center

China Lake, California 93555

$$
{ }^{147} \mathrm{Pm}(\mathrm{B})
$$

199 United States Public Health Service National Institutes of Health

Isotope Laboratory

Building 21

Bethesda, Maryland 20014

CS K 0$), \operatorname{MCP}(0),{ }^{127} \mathrm{Xe}\left(\mathrm{B}^{*}\right)$
200 University of Arizona

P.O. Drawer 4100

Tucson, Arizona 85721

${ }^{47} \mathrm{Ca}(0)$

201 University of Arkansas

Business Manager's Office

Purchasing Section \& 321 Admin. Bldg.

Fayetteville, Arkansas 72701

${ }^{252} \mathrm{Cf}(\mathrm{S}),{ }^{237} \mathrm{~Np}(0), \operatorname{Tgs}(0)$, $235 \mathrm{u}(0)$

202 University of California

Donner Laboratory

Berkeley, California 94720

${ }^{82} \mathrm{Sr}(\mathrm{L})$

203 University of California

P.0. Box 109

La Jolla, California 92037

103 $\mathrm{Ru}(0)$

204 University of California

405 Hilgard Avenue

Los Angeles, California 90024

${ }^{47} \mathrm{Ca}(0), \operatorname{MCP}(0)$

205 University of California Lawrence Berke 1ey Laboratory

Berkeley, California 94701

${ }^{249} \mathrm{Bk}(0),{ }^{249} \mathrm{Cf}(0),{ }^{236} \mathrm{U}(0)$, $235_{U}(B),{ }^{90} \mathrm{Sr}(\mathrm{B})$

206 University of California Lawrence Livermore Laboratory Box 808

Livemore, California 94550

$241_{\mathrm{Am}}(0),{ }^{249} \mathrm{Bk}(0), \quad$ Cap $(0)$ $244 \mathrm{Cm}(0), 246 \mathrm{Cm}(0), 25 \mathrm{Fm}^{2}$ (0), 3 H 390$)$, $237_{\text {Ng }}(0), 231_{\text {P }}(0)$, $239 \mathrm{Pu}(0),{ }^{244} \mathrm{Pu}(0),{ }^{145} \mathrm{Sm}(\mathrm{L})$, ${ }^{146} \mathrm{Sm}(\mathrm{L}),{ }^{99} \mathrm{Tc}(0), \operatorname{Tgs}(0)$, 232 Th $(0), 165_{\mathrm{Ho}(\mathrm{B}), \mathrm{DU}(\mathrm{P})}$

207 University of California

Los Alamos Scientific Laboratory P.O. Box 1663

Los Alamos, New Mexico 87545

${ }^{241} \mathrm{Am}(0) \quad 243_{\mathrm{Am}(0)} \quad 77_{\mathrm{Br}}(\mathrm{L})$, ${ }^{14} \mathrm{C}(\mathrm{O}),{ }^{252} \mathrm{Cf}(\mathrm{S}),{ }^{252} \mathrm{Cf}(\mathrm{O})$,

$146,148,150,151,153 \mathrm{Gg}(b)$,

$231_{\mathrm{Pa}}(0), 238 \mathrm{pu}_{\mathrm{u}}(\mathrm{S}), 239_{\mathrm{P}_{\mathrm{u}}(\mathrm{L})}$,

$242 \mathrm{Pu}(0), \mathrm{SS}(0),{ }^{182} \mathrm{Ta}(\mathrm{B})$, ${ }^{99} \mathrm{Tc}(0), \mathrm{Tg}\left\{(0),{ }^{229} \mathrm{Th}(0)\right.$,

${ }^{168} \mathrm{Tm}(\mathrm{L}), \mathrm{2IO}_{\mathrm{PO}}(\mathrm{B}),{ }^{9} \mathrm{MO}_{\mathrm{MO}}(\mathrm{B})$ 
208 University of California at Davis

Sacramento, California 95817

$67 \mathrm{Cu}(\mathrm{L})$

209 University of California

Parnassus Avenue

San Francisco, California 94143

${ }^{47} \mathrm{Ca}(0)$

210 University of Chicago

Office of the Conptroller

1525 E. Hyde Park Boulevard

Chicago, Illinois 60615

${ }^{247} \mathrm{Ca}(0),{ }^{192} \operatorname{Ir}(0),{ }^{89} \mathrm{Sr}(0)$

211 University of Chicago

Physical Science Department

5641 S. Ingleside Avenue

Chicago, Illinois 60637

$$
\operatorname{CSI}(A),{ }^{68}{ }_{G e}(L)
$$

212 University of Connecticut

U-114

Centra1 Warehouse

Storrs, Connecticut 06268

\section{$28_{\mathrm{Mg}}\left(B^{*}\right)$}

213 University of Delaware

Newark, Delaware 19711

$s S(0),{ }^{230} \mathrm{Th}(0)$

214 University of Kansas Medical Center Kansas City, Kansas 66103

\section{$77_{\mathrm{Br}}(\mathrm{L})$}

215 University of Kentucky

Lexington, Kentucky 40506

$\operatorname{CSI}(0)$

216 University of Massachusetts

Amherst, Massachusetts 01003

$77_{B r}(L)$

217 University of Miami

P.O. Box 8066

Coral Gables, Florida 33146

${ }^{237} \mathrm{~Np}(0),{ }^{230} \mathrm{Th}(0), 233_{\mathrm{U}}(0)$

218 University of Michigan

Ann Arbor, Michigan 48109

$\operatorname{CSI}(0), \operatorname{Tgs}(0),{ }^{3} H(B)$
219 University of New Mexico

Albuquerque, New Mexico 87103

$S S(0)$

220 University of New Mexico

Medical Department

Albuquerque, New Mexico 87131

${ }^{77} \mathrm{Br}(\mathrm{L}),{ }^{65} \mathrm{Cu}(\mathrm{L})$

221 University of Pennsylvania

3025 Walnut Street

Philadelphia, Pennsylvania 19104

${ }^{47} \mathrm{Ca}(0),{ }^{28} \mathrm{Mg}\left(\mathrm{B}^{*}\right)$

222 University of Pennsylvania Hospital

Radiology Department

3400 Spruce Street

Philadelphia, Pennsylvania 19104

${ }^{18} \mathrm{~F}(\mathrm{~B}),{ }^{52} \mathrm{Fe}(\mathrm{B}),{ }^{123} \mathrm{I}(\mathrm{B})$, $28 \mathrm{Mg}\left(B^{\star}\right)$

223 University of Pittsburgh

Pittsburgh, Pennsylvania 15213

$235 \mathrm{u}(0)$

224 University of Rochester Medical Center Health Physics

575 Elwood Avenue

Rochester, New York 14642

RSI (B)

225 University of Tennessee

Knoxville, Tennessee 37916

${ }^{192} \operatorname{Ir}(0)$

226 University of Tennessee - DOE

Agriculture Research Laboratory 1299 Bethel Valley Road

Oak Ridge, Tennessee 37830

$241_{A m}(0), \operatorname{Cap}(0)$

227 University of Texas

Nuclear Medicine Division

Galveston, Texas 77550

${ }^{47} \mathrm{Ca}(0),{ }^{127} \mathrm{Xe}\left(\mathrm{B}^{*}\right)$

228 University of Texas at Dallas

P.O. Box 30365

Dallas, Texas 75230

${ }^{47} \mathrm{Ca}(0)$ 
University of Utah

Radiobiology Division

Bldg. 522

Salt Lake City, Utah 84112

$241_{A m(P), P u(P)}$

230 University of Virginia

Radiaton Safety Officer

RMMF, 12411 th Street, S.W.

Charlottesville, Virginia 22903

$127 \mathrm{Xe}\left(\mathrm{B}^{*}\right)$

231 University of Washington

400 Administration Building

Seattle, Washington 98105

${ }^{229} \mathrm{Th}(0),{ }^{236} \mathrm{U}(0)$

232 University of Wisconsin

COR Safety

1410 Johnson Drive

Madison, Wisconsin 53706

$28_{\mathrm{Mg}}\left(\mathrm{B}^{*}\right)$

233 University of Wisconsin 750 University Avenue Madison, Wisconsin 53706

$3_{H}(0)$

234 U.S. Testing Company, Inc. 2800 George Washington Way Richland, Washington 99352

$241_{A m(0)}$

235 Unitrode Corporation

580 Pleasant Street

Watertown, Massachusetts 01970

$85_{K r(0)}$

236 Varian Instrument Division

Aerograph Operations

2700 Mitchell Drive

Walnut Creek, California

${ }^{63} \mathrm{Ni}(\mathrm{B})$

237 Veterans Administration

San Diego, California 92161

$172 \mathrm{~L} a(L)$

238 Veterans Administration Calm 361

Data Processing Center 1615 Woodward Street East

Austin, Texas 78772

${ }^{47} \mathrm{Ca}(0)$
Veterans Administration Hospital

Chief, Supply Service

Bldg. 1, Room 6137

Hines, lllinois 60141

${ }^{28} \mathrm{Mg}\left(B^{*}\right), \operatorname{CSI}(A),{ }^{47} \mathrm{Ca}(0)$

240 Veterans Administration Hospital

Chief, Supply Service

11201 Benton Street

Lorna Linda, California 92357

$127 \times e(B *)$

241 Veterans Administration Hospital Northport, New York 11768

$127 \mathrm{xe}\left(\mathrm{B}^{\star}\right)$

242 Veterans Administration Hospital

Chief of Supply

1440 V.F.W. Parkway

West Roxbury, Massachusetts 02132

$127 \mathrm{Xe}\left(B^{*}\right)$

243 Veterans Administration Hospital

Nuclear Medicine

1400 V.F.W. Parkway

West Roxbury, Massachusetts 02132

$127 \mathrm{Xe}\left(\mathrm{B}^{*}\right)$

244 Victoreen Inc

10101 Woodland Ave.

Cleveland, Ohio 44104

$\operatorname{DU}(P)$

245 Washington University

St. Louis, Missouri 63110

$77 \mathrm{Br}(\mathrm{L}),{ }^{68} \mathrm{Ge}(\mathrm{L})$

246 Western Electric Company, Inc.

P.0. Box 1409

Lawrence, Massachusetts 01842

$85_{K r}(0)$

247 Western Electric Company, Inc.

777 North Blue Parkway

Lee's Summit, Missouri 64063

$85 \mathrm{kr}(0)$

248 Westinghouse Electric Corporation P.0. Box 284

Elmira, New York 14902

$234 \mathrm{U}(0)$ 
249 Westinghouse Electric Corporation Bettis Atomic Power Laboratory P.0. Box 79

West Mifflin, Pennsylvania 15122

$E U(P), \operatorname{Th}(P)$

250 Westinghouse Hanford Company P.O. Box 1970

Richland, Washington 99352

${ }^{252} \mathrm{Cf}(0),{ }^{85} \mathrm{Kr}(0),{ }_{\mathrm{g}} \mathrm{S}(0)$ ${ }^{237} \mathrm{~Np}(0)$ Tgs(O), $90 \mathrm{SrY}_{\mathrm{Sr}}(\mathrm{H})$, ${ }^{3} \mathrm{H}(\mathrm{H}),{ }^{14} \mathrm{C}(\mathrm{H}),{ }^{239} \mathrm{Pu}(\mathrm{H})$
251 William Beaumont Hospital Nuclear Medical Department 3601 West 13 Mile Road

Royal Oaks, Michigan 48072

$127 \times e\left(B^{*}\right)$

252 Yale University

20 Ashmun Street

New Haven, Connecticut 06520

$103_{R u(0)}$

253 Zirlog, Incorporated

10460 Bubb Road

Cupertino, California 95014

$85_{K r}(0)$ 


\section{FOREIGN COUNTRIES}

Australia

254 Australian Atomic Energy Commission Research Establishment

Lucas Heights, NSW

AUSTRALIA

$$
127 \times \mathrm{e}\left(\mathrm{B}^{\star}\right)
$$

255 Embassy of Australia

1601 Massachusetts Avenue, N.W.

Washington, D.C. 20036

$230 \operatorname{Th}(0), 234 \mathrm{U}(0), 235 \mathrm{U}(0)$, $236 \mathrm{U}(0), 238_{U}(0)$

Austria

256 International Atomic Energy Agency Kaerntnerring $11 \& 13$

A \& 1010 Vienna, AUSTRIA

$237 \mathrm{~Np}(0), 2 \operatorname{Tgs}(0),{ }^{232} \mathrm{Th}(0)$,

\section{Belgium}

257 Central Bureau for Nuclear Measurements of the European Communities

B \& 2440 Geel, Steenweg Maar Retie BELGIUM $243 \mathrm{Am}(0), \operatorname{MCP}(0), \operatorname{Tgs}(0)$,
$230_{\mathrm{Th}(0)}, 235_{\mathrm{U}(0)}$

258 Centre D'etude de L'energie Nucleaire 144 Avenue Plasky

Brussels 4, BELGIUM

$242 p_{u}(0)$

Canada

259 Atomic Energy of Canada Limited Chalk River, Ontario, CANADA

${ }^{3} H(0),{ }^{230} \operatorname{Th}(0)$

260 Atomic Energy of Canada, Ltd. Chalk River Nuclear Laboratories

Chalk River, Ontario, CANADA

$\operatorname{CSI}(A)$

261 Atomic Energy of Canada, Ltd.

Whiteshell Nuclear Research Establishment

Pinawa, Manitoba, CANADA

$C_{\text {ap }}(0), 231_{p a(0)}, 234_{U}(0)$
262 Atomic Energy of Canada, Ltd.

P.0. Box 6300

Postal Station J

Ottawa, CANADA

$33 p(0)$

263 Carleton University

Accounts Payable Department

Business Office

301 Administration Bldg.

Ottawa, Ontario

K1 S 5 B6

CANADA

${ }^{230} \operatorname{Th}(0)$

264 Hospital Saints \& Lac Achats

1058 St. \& Oenis

Montreal, P.0.

$\mathrm{H} 2 \times 3 \quad 3 \quad J 4$

CANADA

${ }^{47} \mathrm{Ca}(\mathrm{O})$

265 MCGill University

Montreal 2, P.G.

CANADA

$235 \cup(0)$

266 McMaster University Medical Center

Forsyth Avenue Receiving

Hami1ton, Ontario

CANADA

$127 \mathrm{Xe}\left(\mathrm{B}^{*}\right)$

267 University Hospital

Nuclear Medicine Department

339 Windermere Road

London, Ontario

CANADA

$127 \times \mathrm{e}\left(B^{*}\right)$

268 University of Alberta

Edmonton, Alberta

CANADA

${ }^{47} \mathrm{Ca}(0)$

269 University of British Columbia Vancouver 8, British Columbia CANADA

$3_{H(0)}$

270 University of Toronto

Toronto, Ontario

CANADA

${ }^{252} \mathrm{Cf}(0)$ 
Denmark

271 Isotope Pharmacy

378 Frederiksundsvej

Broenshoej

DENMARK

$127 \times \mathrm{e}\left(\mathrm{B}^{*}\right)$

Federal Republic of Germany

272 Bonn Universitat

Nussallee 12 \& 14

55 Bonn,

FEDERAL REPUBLIC OF GERMANY

$$
{ }^{230} \operatorname{Th}(0)
$$

273 Buchler and Company

Franfurter Str. 294

Braunschweig,

FEDERAL REPUBLIC OF GERMANY

${ }^{37} \mathrm{Ar}(0),{ }^{129} \mathrm{I}(0),{ }^{95_{\mathrm{Zr}}(0)}$

274 Darmstadt, Technischen Hochschule 61 Darmstadt,

FEDERAL REPUBLIC OF GERMANY

$242 p u(0)$

275 EURATOM Transuranium Institute

P.O. Box 452

75 Karlsruhe,

FEDERAL REPUBLIC OF GERMANY

${ }^{239} p_{u}(0),{ }^{244} p_{u}(0),{ }^{252} \mathrm{Cf}(\mathrm{S})$

276 Freie Universitat Berlin

Fachbereich Physik

FB20, WE1A

Boltzmannstrasse 20

1000 Berlin 33

P. West

FEDERAL REPUBLIC OF GERMANY

$127 \times \mathrm{e}\left(B^{*}\right)$

277 Gesellschaft Fur Kernforschung Mbh

Institute Fuer Angewandte

Reaktor Physik

Weverstrasse 5

$75 \mathrm{Karls} r u h e$

FEDERAL REPUBLIC OF GERMANY

$241_{A m}(0),{ }^{243} A m(0), C a p(0)$, 238pu(0)

278 Geissen Universitat

6300 Giessen

FEDERAL REPUBLIC OF GERMANY

${ }^{244} p_{u}(0)$
279 Kernkraftwerk Obrigheim MBH

6951 Obrigheimam Neckar

Frankfurt

FEDERAL REPUBLIC OF GERMANY

$234 v(0)$

280 Kommission Der Europaischen Germeinschaften

Germeinsame Forschungsstelle

Reaktorgelande 07514

Lepo1dshafen

FEDERAL REPUBLIC OF GERMANY

${ }^{252} \mathrm{Cf}(\mathrm{S})$

281 Universitat Dortmund

Postfach D 0 \& Hombrach 500

4600 Dortmund \& Eichlinghofen

FEDERAL REPUBLIC OF GERMANY

${ }^{99} \mathrm{Tc}(0)$

282 University of Eremen

Zentraler Einkauf

280 Bremen

FEDERAL REPUBLIC OF GERMANY

$127 \mathrm{Xe}\left(\mathrm{B}^{*}\right)$

\section{France}

283 CEA

Department Des Radioelements

B.P. No. 2

91190 G if Sur Yvette

FRANCE

${ }^{95} \mathrm{Nb}(0),{ }^{147} \mathrm{Pm}_{\mathrm{m}}(0), 240 \mathrm{pu}_{\mathrm{u}}(0)$,

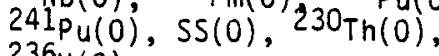
$236 U(0)$

284 Centre d'Etudes Nuclearies de Sontenay-Aux-Roses 92260 Sontenay-Aux-Roses FRANCE

${ }^{252} \mathrm{Cf}(\mathrm{S})$

Great Britain

285 Amersham Bucks

Radiochemical Center, Ltd.

Bldg. 250, Cargo Terminal

London, Heathrow Airport

GREAT BRITAIN

$127 \mathrm{Xe}\left(\mathrm{B}^{*}\right),{ }^{252} \mathrm{Cf}(\mathrm{S})$ 
286 Amersham International Limited

White Lion Road Amersham

Buckinghamshir e Ęngland

H P7 9 LL

GREAT BRITAIN

${ }^{144} \mathrm{Ce}(0),{ }^{244} \mathrm{Cm}(0),{ }^{137} \mathrm{Cs}(0)$,

$55 \mathrm{Fe}(0), 3 \mathrm{H}(0),{ }^{129} \mathrm{I}(0)$,

${ }^{85} \mathrm{Kr}(0), \operatorname{MCP}(0),{ }^{147} \mathrm{Pm}_{\mathrm{m}(0)}, \mathrm{RW}(0)$,

${ }^{99} \mathrm{Tc}(0), \operatorname{Tgs}(0), 127 \mathrm{Xe}\left(\mathrm{B}^{*}\right)$,

$95 \mathrm{Zr}(0)$

287 Brandhust Co. Ltd.

Wel1 ington Road

High Wycombe, Bucks

H P 123 P S England

GREAT BRITAIN

$3_{H}(0)$

288 Hammersmith Hospital

Ducane Road

London, W. 12, England

GREAT BRITAIN

${ }^{82} \mathrm{Sr}(\mathrm{L}),{ }^{242 \mathrm{pu}(0)}$

289 Mallinckrodt, Ltd.

81dg. $521129 \mathrm{G}$

Southampton Road

Middlesex

GREAT BRITAIN

\section{$127 \mathrm{Xe}\left(B^{*}\right)$}

290 Saunders \& Roe Developments, Ltd.

North Hyde Road

Hayes, Middlesex, England

GREAT BRITAIN

$3_{H}(0)$

291 United Kingdom Atomic Energy Establishment

Harwell, Didcot, Berkshire

England

GREAT BRITAIN

$\operatorname{MCP}(0),{ }^{242} \mathrm{Pu}(0)$

292 University of Leeds

Leeds 2, England

GREAT BRITAIN

${ }^{242} \mathrm{p}_{\mathrm{u}}(0$

Hong Kong

293 Semi \& Conductor Devices, Ltd.

$59 \& 61$ Wong Chuk Hang Road

Aberdeen, Hong Kong

$85_{k r}(0)$
Japan

294 Cancer Institute Hospital

Tokyo,

JAPAN

${ }^{252} \mathrm{Cf}(\mathrm{S})$

295 Gakushu in University

Faculty of Science

1-5-1 Mejiro, Toshima - Ku

Tokyo, 171

JAPAN

$11_{B(B)}$

296 Japan Atomic Energy Research Institute

Tokai Research Establishment

Tokai \& Mura, Naka \& Gun JAPAN

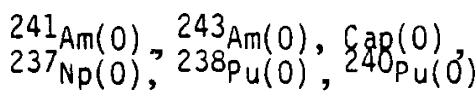

297 Japan Radioisotope Association

Hon \& Komagome 2 Chome 28

Bunkyo \& $\mathrm{Kle}$

Tokyo

JAPAN

${ }^{3} \mathrm{H}(0)$

298 Power Reactor and Nuclear Fuel Development Corpor $9 \& 13$

I \& Chome, Akasake, Minator \& Ku

Tokyo

JAPAN

$242 p u(0), 233 u(0)$

299 University of Tokyo

Solid State Physics

Roppongi, Minato-ku

Tokyo

JAPAN

$60_{N i(B)}$

The Netherlands

300 N. V. Philips \& Duphar

P.O. Box 2

Weesp

THE NETHERLANDS

$103_{R u(0), ~} S S(0),{ }^{99} T c(0)$

New Zealand

301 New Zealand Embassy

1145 \& 19th Street

Washington, D.C. 20036

$\operatorname{CSI}(0)$ 
Norway

302

Institute for Atomic Energy

Isotope Laboratories

$\mathrm{N}-2007$

Kjeller

NORWAY

$127 \mathrm{Xe}\left(\mathrm{B}^{*}\right)$

People's Republic of China

303 China National Institute Import \& Export Corporation Beijing

PEOPLE'S REPUBLIC OF CHINA

$3_{H}(B)$

Portuga1

304 Junta de Energia Nuclear

Laboratorio de Fisica e Engenharea Nucleares

Estrada Nacional No. 10

Sacavem

PORTUGAL

${ }^{233} U(0), 235 U(0)$

Republic of China

305 Coordination Council for North American

Affairs

133 PO \& AI Road

Taipei, Taiwan

REPUBLIC OF CHINA

${ }^{242} \mathrm{pu}(0)$, SS, ${ }^{233} \mathrm{u}(0)$

Republic of Ireland

306 Electramed - Ireland, Ltd.

c/o Aer Lingus Import Cargo

143 Phibsboro Road

Dublin

REPUBLIC OF IRELAND

$28 \mathrm{Mg}\left(B^{*}\right)$

Switzerland

307 EIDG, Institut fur Reaktor forschung

Wurenlingen

SWITZERLAND

${ }^{3} \mathrm{H}(0), M C P 147 P_{m}(0),{ }^{99} \mathrm{~T} c(0)$
308 Geneva Research Centres

7. route de Drize

1227 Carouge

Geneva

SWITZERLAND

${ }^{14} \mathrm{C}(B)$

309 Schweizeriches Institut fur Nuklearforschung

Neunbrunnenstrasse 85 ,

8050 Zurich, Switzerland

MB \& Microtec, Incorporated

frieburgstrasse 624

$\mathrm{CH} \& 3172$ Nlederwangen/Berne

SWITZERLAND

$3_{H}(0)$

Union of South Africa

310 Chemlab

Johannesburg

UNION OF SOUTH AFRICA

$127 \mathrm{Xe}\left(\mathrm{B}^{*}\right)$

311 Tygerberg Hospital

Nuclear Medicine Department

Parowvalle i

Cape Province

UNION OF SOUTH AFRICA

$127 \mathrm{Xe}\left(\mathrm{B}^{\star}\right)$

312 Well Organization c/O P TY Limited

P.O. Box 31315

Braamfontein 2017

Johannesburg

UNION OF SOUTH AFRICA

$127 \mathrm{Xe}\left(B^{*}\right)$ 


\subsection{ALPHABETICAL LIST OF ISOTOPES CROSS-REFERENCED TO CUSTOMER NUMBER}

Americium (Am) 241

$1,11,14,25,36,48,61101,108$,

$116,118,126,171,177,206,207$,

$226, \quad 229,234,277,296$

Americium (Am) 243

$1,73,120,135,179,207,257,277$, 296

Argon (Ar) 37

176, 273

Arsenic (As) 73

12

Astatine (At) 211

62

Barium (8a) 128

109

Barium (Ba) 133

11, 169

$\operatorname{Barium}(\mathrm{Ba}) 140$

1

Berkelium (8x) 249

8, 205, 206

Boron (8) 11

295

Bromine (Br) 77

$15,56,78,107,207,214,216,220$,

245

Cadium (Cd) 109

109

Calcium (Ca) 47

$31,32,67,76,83,89,90,91,97$

$128,171,185,188,200,204,209$,

$221,227,228,238,239,264,268$

Calcium (Ca) 147

165
Calcium (Ca) 247

210

Californium (CF) 249

8,205

Californium (Cf) 252

$1,20,35,51,73,101,125,171,184$,

$189,201,207,250,279,275,280$,

$284, \quad 285,294$

Carbon (C) 14

$66,108,121,207,250,308$

Cerium (Ce) 144

$1,19,50,73,108,171,286$

Cesium (Cs) 134

11

Cesium (Cs) 137

$11,18,50,61,65,72,73,98,108$,

$126,137,147,169,171,286$

Cobalt (Co) 57

169

Cobalt (Co) 58

109

Cobalt (Co) 60

$1,11,44,95,106 a, 169$

Copper (Cu) 65

220

Copper (Cu) 67

16, 208

Curium (Cm) 243

. 84

Curium (Cm) 244

$1,11,14,135,171,206,286$

Curium (Cm) 246

206 
Curium (Cm) 248

35

Cyclotron Service Irradiations (CSI)

$26, \quad 65,73,85,86, \quad 108,120,171$,

$172, \quad 199,211,215,218,239,260,301$

Depleted Uranium (DU)

$180,206,244$

Ensteinium (Es) 253

$8, \quad 207$

Encapsulation (Cap)

$8,14,36,48,61,101,108,116,118$,

$120,171,181,206,226,261,277,296$

Enriched Uranium (EU)

189, 249

Europium (Eu) 152

8, $19, \quad 137$

Fermium (Fm) 257

206

Fluorine (F) 18

62, 89, 191, 222

Gadolinium (Gd) 146, 148, 150, 151

207

Gadolinium (Gd) 153

$1, \quad 4,50,61,65, \quad 108,172,207$

Germanium (Ge) 68

$14,89,109,171,192,211,245$

God 1 (Au) 195

109

Hafnium (Hf) 122

171

Hafnium (Hf) 172

22
Holmium (Ho) 165

206

Indium (In) 114

172

Iodine (I) 123

$56,78,222$

lodine (I) 129

$38,71,73,81, \quad 108,189,273,286$

lodine (I) 131

171

Iridium (Ir) 192

$1,4,10,47,61,68,71,153,162$, $172, \quad 210, \quad 225$

Iron $(\mathrm{Fe}) 52$

138, 222

Iron $(\mathrm{Fe}) 55$

$73,108,286$

Iron $(\mathrm{Fe}) 59$

8

Krypton $(\mathrm{Kr}) 85$

$2,3,4,5,6,29,30,33,43,46,49$, $52, \quad 54, \quad 55, \quad 59, \quad 63, \quad 64, \quad 69, \quad 73, \quad 75$,

$92,98,100,103,105,106,108,124$,

$125,127,129,130,131,141,146$,

$148,149,150,151,155,161,167$,

$168,170,171,187,195,235,246$,

$247, \quad 250, \quad 233, \quad 286, \quad 293$

Lanthanum (La) 172

237

Lutetium (Lu) 172

22

Magnesium (Mg) 28

$21,115,133,212,221,222,232,239$, 306

Manganese (Mn) 59

11 
Miscel 1aneous Compounds (MCP)

$50,70, \quad 71, \quad 71,74,77,98, \quad 104, \quad 147$,

$154, \quad 163,171,172,199,204,257$,

286, 291, 307

Molybdenum (Mo) 93

207

Natural Uranium (NU)

$11,166,171$

Neptunium (Np) 237

$1,8, \quad 45,111,132,143,144,171$,

$183, \quad 189,201,206,217,250,256,296$

Nickel (Ni) 60

299

Nickel (Ni) 63

236

Niobium (Nb) 95

98, 283

Osmium (Os) 191

23

Palladium (Pd) 109

27

Phosphorus (P) 33

$65,108,262$

Plutonium ( $\mathrm{Pu}$ )

$180,182,229$

Plutonium (Pu) 236

$1,13,73, \quad 178, \quad 181,189$

Plutonium (Pu) 237

11

Plutonium (Pu) 238

11, 101, 181, 186, 207, 277, 296

Plutonium (Pu) 239

$1,8,11,73,117,171,183,206,207$, $250, \quad 275$
Plutonium (Pu) 240

283, 296

Plutonium (Pu) 241

283

Plutonium (Pu) 242

$1,88,136,171,207,358,274,288$,

$291, \quad 292, \quad 298, \quad 305$

Plutonium (Pu) 244

206, $275, \quad 278$

Polonium (Po) 208

73

Polonium (Po) 210

11, 207

Potassium (K) 43

93

Promethium (Pm) 147

$11,14,65,70,172,175,198,283$, $286, \quad 307$

Protactinium (Pa) 231

73, 206, 207, 261

Reactor Services Irradiations (RSI)

$8,40,42,51,53,110,112,133,139$, $159,188,224$

Ruthenium (Ru) 97

11, 171

Ruthenium (Ru) 103

1, 29, 152, 203, 252, 300

Ruthenium (Ru) 106

$8, \quad 65,73,137$

Samarium (Sm) 145

206

Samarium (Sm) 146

206 
Samarium (Sm) 151

19, 108

Selenium ( $\mathrm{Se}$ ) 75

$65, \quad 86, \quad 108, \quad 156, \quad 171,174$

Silver (Ag) 110

137, 169

Sodium ( $\mathrm{Na}) 22$

$39, \quad 109, \quad 171$

Special Services (SS)

$1,5,14,35,50,60,66,84,86,108$, $125, \quad 162, \quad 171, \quad 172,189,207,213$,

$219, \quad 250,283, \quad 300,305$

Strontium (Sr) 82

104, 152, 157, 192, 202, 288

Strontium (Sr) 89

$28,29,37,57,60,65,73,121,158$,

Strontium (Sr) 90

11, 65, 73, 137, 163, 169, 175, 205, 250

Strontium (Sr) $90-$ Yttrium (Y)

$11,137,169,250$

Tantalum (Ta) 182

$71, \quad 207$

Target Preparations (Tgs)

$8,9,11,14,20,73,111,117,119$, $126, \quad 132, \quad 143,144,171,189,201$, $206,207,218,250,256,257,286$

Technetium (Tc) 99

$108, \quad 128,137,156,158,171,185$,

$189,206,207,281, \quad 286, \quad 300,307$

Thorium (Th)

$171, \quad 249$

Thorium (Th) 229

$14, \quad 207,231$
Thorium (Th) 230

1, a, 73, 213, 217, 255, 257, 259, $263,272, \quad 283$

Thorium (Th) 232

a, 50, 171, 206, 256

Thulium (Tm) 168

207

Tritium (H) 3

11, 14, 21, 34, 41, 59, 65, 79, 80, $82,90,102,108,116,122,140,142$, $145,167,171,189,206,218,233$, $250,259,269,286,287,290,297$, $303,307,309$

Tungsten (W) 178

109

Tungsten (W) 181

12

Uranium (U) 233

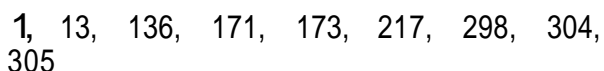

Uranium (U) 234

$71,99, \quad 134,248, \quad 255,261,279$

Uranium (U) 235

$8, \quad 13,15,71,73,94,119,132,171$,

$201,205,223,255,256,257,265,304$

Uranium (U) 236

$71,73,123,173,205,231,255,283$

Uranium (U) 238

$8, \quad 9, \quad 71, \quad 132, \quad 171, \quad 255,256$

Vanadium (V) 48

17

Vanadium (V) 49

197

Withdrawn Isotopes (RW)

14,286 
Xenon (Xe) 127

$7,14,15,24,58,73,86,87,96$, $113,114,160,190,192,193,194$, $196,199,227,230,240,241,242$,

$243,151,254,266,267,271,276$,

$282,285,286,289,302,310,311,312$
Yttrium (Y) 88

109

Zirconium ( $\mathrm{Zr}$ ) 95

$1,8,60,73,108,273,286$ 
28

8 


\section{GEOGRAPHICAL LOCATION OF RADIOISOTOPE CUSTOMERS \\ (identified by customer numbers)}

\section{STATES}

Arizona (AZ)

$5, \quad 55,103,105,200$

Arkansas (AR)

201

California $(C A)$

$15, \quad 16,17, \quad 18,22,43,46,50,51$,

$54,60,63,68,69,70,73,75,82$,

$83,92,100,102,106,119,130,132$,

$144,146,147,149,150,151,154$,

161. 168. 176., 196. 198., 202., 203.

$204,205,206,208 ; 209,236,237$;

$240, \quad 253$

Colorado (CO)

$25, \quad 79, \quad 136, \quad 182,183$

Connecticut (CT)

212, 252

Delaware

213

District of Columbia (OC)

$19, \quad 56,57, \quad 185, \quad 189, \quad 197,255,301$

Florida ( $F L)$

$30, \quad 45, \quad 94, \quad 186,217$

Georgia (GA)

7, 58

Idaho (ID)

$1,9,40,42$

Illinois (IL)

$4,8,44,85,97,138,179,180,210$, $211, \quad 239$

Indiana (IN)

$66,67,96,124,128$

lowa (IA)

71
Kansas

214

Kentucky (KY)

173, 215

Louisiana (LA)

$47, \quad 153,177$

Maryland (MD)

$77,78,106 \mathrm{a}, 143,163,187,190,191$, 192, 194,199

Massachusetts (MA)

$6,23,24,62,89,90,107,108,109$, $155, \quad 162,216,235,242,243,246$

Michigan (MI)

$34,80,218,251$

Minnesota (MN)

91,98

Missouri (MO)

$76,86,87, \quad 245,247$

Nebraska (NB)

32

Nevada (NV)

135

New Jersey (NJ)

$2,3,12,33,41,59,72,88,95,117$, $125,126,131,139,156,157,164,195$

New Mexico (NM)

$84,141,207,219,220$

New York (NY)

$14, \quad 26, \quad 27,31,36,38,53,81,99$,

$104,110,111,116,133,142,145$,

$152,158,159,160,174,175,178$,

$224, \quad 241, \quad 248$

North Carolina (NC)

$37,113,114$ 
,Ohio (OH)

$21,65,74,101,115,121,122,127$, 134,244

Oklahoma (OK)

29

Oregon (OR)

123

Pennsylvania (PA)

$10,20,64,129,140,221,222,223$, 249

South Carolina (SC)

35, 193

Tennessee (TN)

$120, \quad 170,171,172,225,226$

Texas (TX)

$28,48,61,112,118,165,166,167$, $188,227,228,238$

Utah (UT)

148,229

Virginia (VA)

$39, \quad 49,52, \quad 184,230$

Washington (WA)

$11,13,137,169,181,231,234,250$

Wisconsin (WI)

$93,232,233$

FOREIGN

Australia

254,255

Austria

256

Be $1 \mathrm{~g}$ ium

$257, \quad 258$
Canada

$259,260,261,262,263,264,265$,

$266,267,268,269,270$

Denmark

271

Federal Republic of Germany

$272, \quad 273,274,275,276,277,278$,

$279,280,281,282$

France

283, 204

Great Britain

$285,286,287,288,289,290,291,292$

Hong Kong

293

Japan

294, 295, 296, 297, 298, 299

The Nether1ands

300

New Zealand

301

Norway

302

People's Republic of China

303

Portugal

304

Republic of China

305

Republic of Ireland

306

Switzerland

$307, \quad 308, \quad 309$

Union of South Africa

$311,311,312$ 
5. RADIOISOTOPE SALES AND TRANSFERS--FY198]

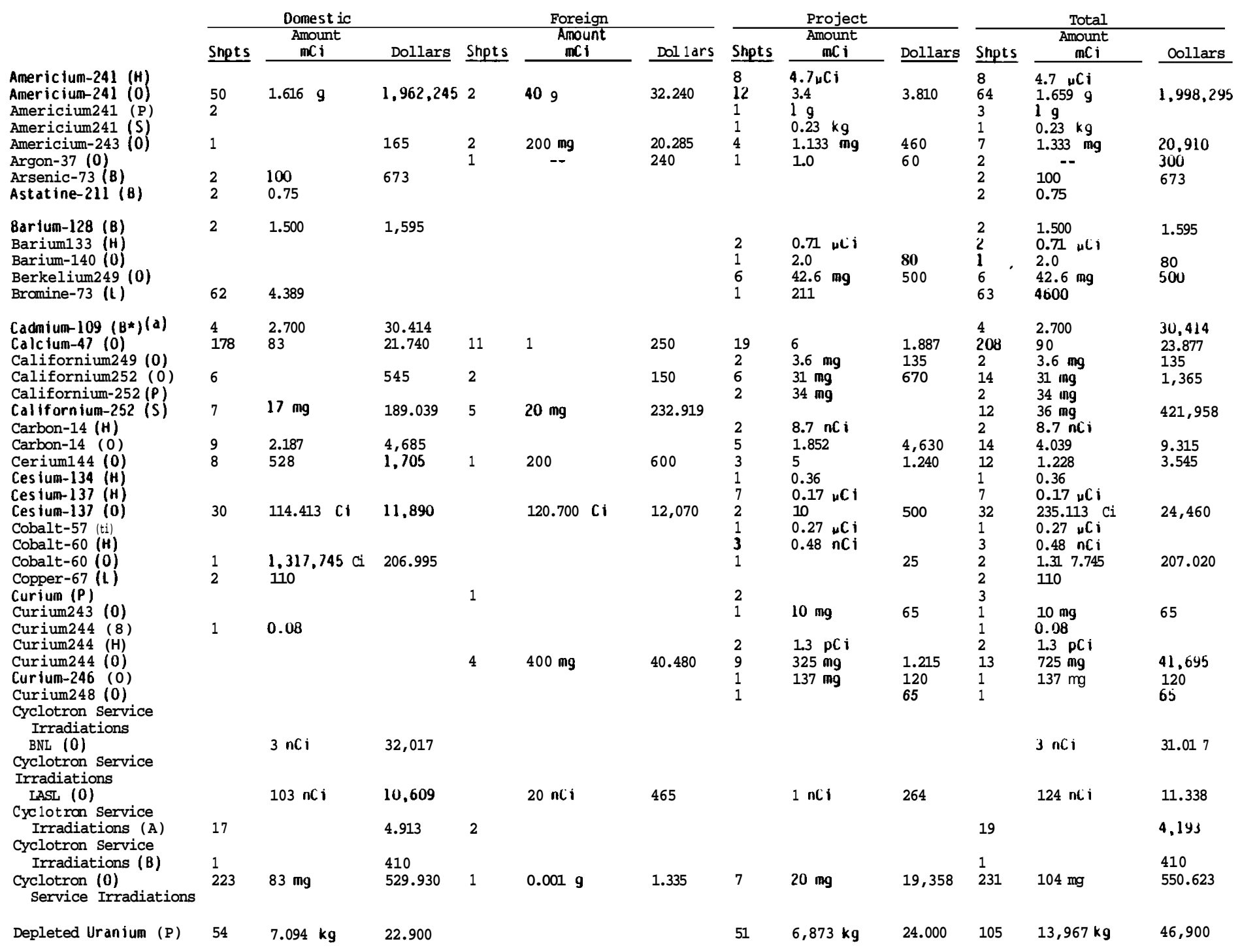




\section{RADIOISOTOPE SALES AND TRANSFERS--FY1981}

Einsteinium253 (0) Encapsulation (0) Enriched Uranium ( $P$ ) $\left.\begin{array}{l}\text { Europium152 (H) } \\ \text { Europium152 } \\ 0\end{array}\right)$

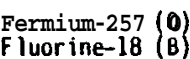

Gadolinium (L) (0)

Germanium68 (L) $(\mathrm{a})$

Gold-195 (6)

Hafnium-172 (L)

I ndium-114 (0)

lodine-129 (0)

Iridiuan-192 (0)

Iridium-192 Meta (0)

Iron-52 (L)

Iron-55 $(0)$

Krypton-85 (0)

Krypton-85 Enriched (0)

Magnesium-28 (B*) (a)

Manganese54 (H)

Miscellaneous Compounds $\left(B^{*}\right)$

Miscellaneous Compounds (B)

Miscellaneous Compounds $(0)$

Natural Uranium (H)

Natural Uranium (0)

Neptunium237 (0)

Neptunium237

Metal (0)

Oxide (0)

Niobium95 (0)

Osmium191 (B)

Palladium-109 (B)

Phosphorus-33 (0)

Plutonium $(P)$

Plutonium237 (O)

\begin{tabular}{|c|c|c|c|c|c|c|c|c|c|c|c|c|}
\hline \multicolumn{3}{|c|}{ Domestic } & \multicolumn{4}{|c|}{ Foreign } & \multicolumn{3}{|c|}{ Project } & \multicolumn{3}{|c|}{ Total } \\
\hline Shpts & $\begin{array}{c}\text { Amount } \\
\mathrm{IC} \mathrm{i} \\
\end{array}$ & Dollars & Shpts & & ount & Dollars & Shpts & $\begin{array}{c}\text { Amount } \\
\mathrm{mC} i\end{array}$ & Dollars & Shpts & $\begin{array}{c}\text { Amount } \\
\mathbf{m C} \mathbf{i}\end{array}$ & Dollars \\
\hline 3 & & 263.041 & $\begin{array}{l}8 \\
3\end{array}$ & 94.666 & g & $\begin{array}{l}51,095 \\
85,000\end{array}$ & $\begin{array}{l}3 \\
67 \\
41\end{array}$ & $\begin{array}{l}2 \mathbf{~ m g} \\
7,377 \mathbf{g}\end{array}$ & $\begin{array}{l}250 \\
49,550 \\
1,600\end{array}$ & $\begin{array}{l}3 \\
75 \\
47\end{array}$ & $\begin{array}{l}2 \mathrm{mg} \\
102.063 \mathrm{~g}\end{array}$ & $\begin{array}{l}250 \\
363,686 \\
66,600\end{array}$ \\
\hline 1 & 2 & 40 & & & & & $\begin{array}{l}1 \\
1\end{array}$ & $\begin{array}{l}1 \\
10\end{array}$ & 200 & $\begin{array}{l}1 \\
2\end{array}$ & $\begin{array}{l}1 \\
12\end{array}$ & 240 \\
\hline 45 & 662 & & & & & & 2 & & 130 & $\begin{array}{l}2 \\
45\end{array}$ & 662 & 130 \\
\hline 14 & $15 \mathrm{Ci}$ & 22,750 & & & & & $\begin{array}{l}1 \\
5 \\
6\end{array}$ & $\begin{array}{l}1500 \\
6 \mathrm{Ci} \\
172\end{array}$ & 9,500 & $\begin{array}{l}1 \\
19 \\
6\end{array}$ & $\begin{array}{l}1500 \\
21 \mathrm{Ci} \\
172\end{array}$ & 32.250 \\
\hline $\begin{array}{l}3 \\
1\end{array}$ & $\begin{array}{l}645 \\
100\end{array}$ & 8,417 & & & & & & & & $\begin{array}{l}3 \\
1\end{array}$ & $\begin{array}{l}645 \\
100\end{array}$ & 8.417 \\
\hline 1 & 1 & & & & & & & & & 1 & 1 & \\
\hline 3 & 104 & & & & & & 1 & 250 & 2500 & $\begin{array}{l}1 \\
3\end{array}$ & $\begin{array}{l}250 \\
104\end{array}$ & 2500 \\
\hline $\begin{array}{l}7 \\
1\end{array}$ & $i^{4.1004}$ & $\begin{array}{l}6.225 \\
25\end{array}$ & 4 & 1.551 & & 3.102 & $\begin{array}{l}2 \\
1\end{array}$ & $\begin{array}{l}0.450 \\
1\end{array}$ & $\begin{array}{l}900 \\
25\end{array}$ & $\frac{13}{2}$ & $\frac{6.1014}{2}$ & $\begin{array}{l}12.227 \\
50\end{array}$ \\
\hline $\begin{array}{l}211 \\
1\end{array}$ & $\frac{1,303,382 \mathrm{Ci}}{20}$ & $1,878,568$ & & & & & 10 & $1.365 \mathrm{Ci}$ & 2.730 & $\begin{array}{l}221 \\
1\end{array}$ & $\frac{1}{20}, 304,747 \mathrm{Ci}$ & $1,881,298$ \\
\hline $\begin{array}{l}2 \\
1\end{array}$ & $\begin{array}{l}630 \\
0.005\end{array}$ & 2,362 & 8 & 16.600 & & 62.250 & & & & $\begin{array}{l}10 \\
1\end{array}$ & $\begin{array}{l}17,230 \\
0.005\end{array}$ & 64.612 \\
\hline $\begin{array}{l}199 \\
9\end{array}$ & $\begin{array}{l}4,922 \mathrm{Ci} \\
1.296 \mathrm{Ci}\end{array}$ & $\begin{array}{l}164,490 \\
190,040\end{array}$ & $\begin{array}{l}7 \\
1\end{array}$ & $\begin{array}{l}286 \mathrm{Ci} \\
50 \mathrm{Ci}\end{array}$ & & $\begin{array}{l}6.292 \\
3,000\end{array}$ & 19 & $534 \mathrm{Ci}$ & 10.361 & $\begin{array}{l}225 \\
11\end{array}$ & $\begin{array}{l}5,742 \mathrm{Ci} \\
1.346 \mathrm{Ci}\end{array}$ & $\begin{array}{l}181.143 \\
193,040\end{array}$ \\
\hline 18 & 6.2 & 3.127 & 4 & 0.64 & & 306 & 2 & $0.28 \mathrm{nCi}$ & & $\begin{array}{l}22 \\
2\end{array}$ & $\begin{array}{l}7.03 \\
0.28 \mathrm{nCi}\end{array}$ & 3,435 \\
\hline $\begin{array}{l}1 \\
23 \\
18\end{array}$ & $6 \mathrm{Ci}$ & $\begin{array}{l}7.742 \\
720 \\
2.142\end{array}$ & $\begin{array}{l}4 \\
1\end{array}$ & $2 \mathrm{nCi}$ & & $\begin{array}{l}120 \\
110\end{array}$ & 6 & $1 \mathbf{n C} \mathbf{i}$ & 269 & $\begin{array}{l}1 \\
27 \\
25\end{array}$ & $207 \mathrm{nCi}$ & $\begin{array}{l}7,742 \\
840 \\
2,521\end{array}$ \\
\hline 1 & $50 \mathrm{~g}$ & 65 & & & & & $\begin{array}{l}4 \\
1\end{array}$ & $0.33 \mathrm{mg}$ & 65 & $\begin{array}{l}4 \\
2\end{array}$ & $\begin{array}{l}0.33 \mathrm{mg} \\
50 \mathrm{~g}\end{array}$ & 130 \\
\hline 3 & $1,133 \mathrm{mg}$ & 531 & 4 & 25.500 & $\mathrm{mg}$ & 7,944 & 4 & $6.064 \mathrm{mg}$ & 2.046 & 11 & $32,697 \mathrm{mg}$ & 10.521 \\
\hline 1 & $500 \mathrm{mg}$ & 65 & & & & & & & & 1 & $500 \mathrm{mg}$ & 65 \\
\hline $\begin{array}{l}3 \\
12\end{array}$ & $\begin{array}{l}6.743 \\
200\end{array}$ & $\begin{array}{l}1.765 \\
8,000\end{array}$ & 1 & $\begin{array}{l}20 \\
1\end{array}$ & & $\begin{array}{l}4.500 \\
40\end{array}$ & 4 & 16.075 & 2,673 & $\begin{array}{l}7 \\
13\end{array}$ & $\begin{array}{l}42,818 \\
201\end{array}$ & $\begin{array}{l}9.138 \\
8.040\end{array}$ \\
\hline 7 & 10,650 & & & & & & & & & 7 & 10.650 & \\
\hline $\begin{array}{l}16 \\
20 \\
2 \\
3\end{array}$ & $\begin{array}{l}6.832 \\
1,839\end{array}$ & $\begin{array}{l}23.325 \\
2.065\end{array}$ & 1 & 1 & & 35 & $\begin{array}{l}33 \\
5\end{array}$ & $\begin{array}{l}610 \mathrm{~g} \\
1\end{array}$ & $\begin{array}{l}26,300 \\
3,489 \\
2,975\end{array}$ & $\begin{array}{l}16 \\
21 \\
35 \\
8\end{array}$ & $\begin{array}{l}6,632 \\
1,840 \\
610 \mathrm{~g} \\
1\end{array}$ & $\begin{array}{l}23.360 \\
26.300 \\
5.554 \\
2.975\end{array}$ \\
\hline
\end{tabular}


5. RADIOISOTOPE SALES AND TRANSFERS--FY1981

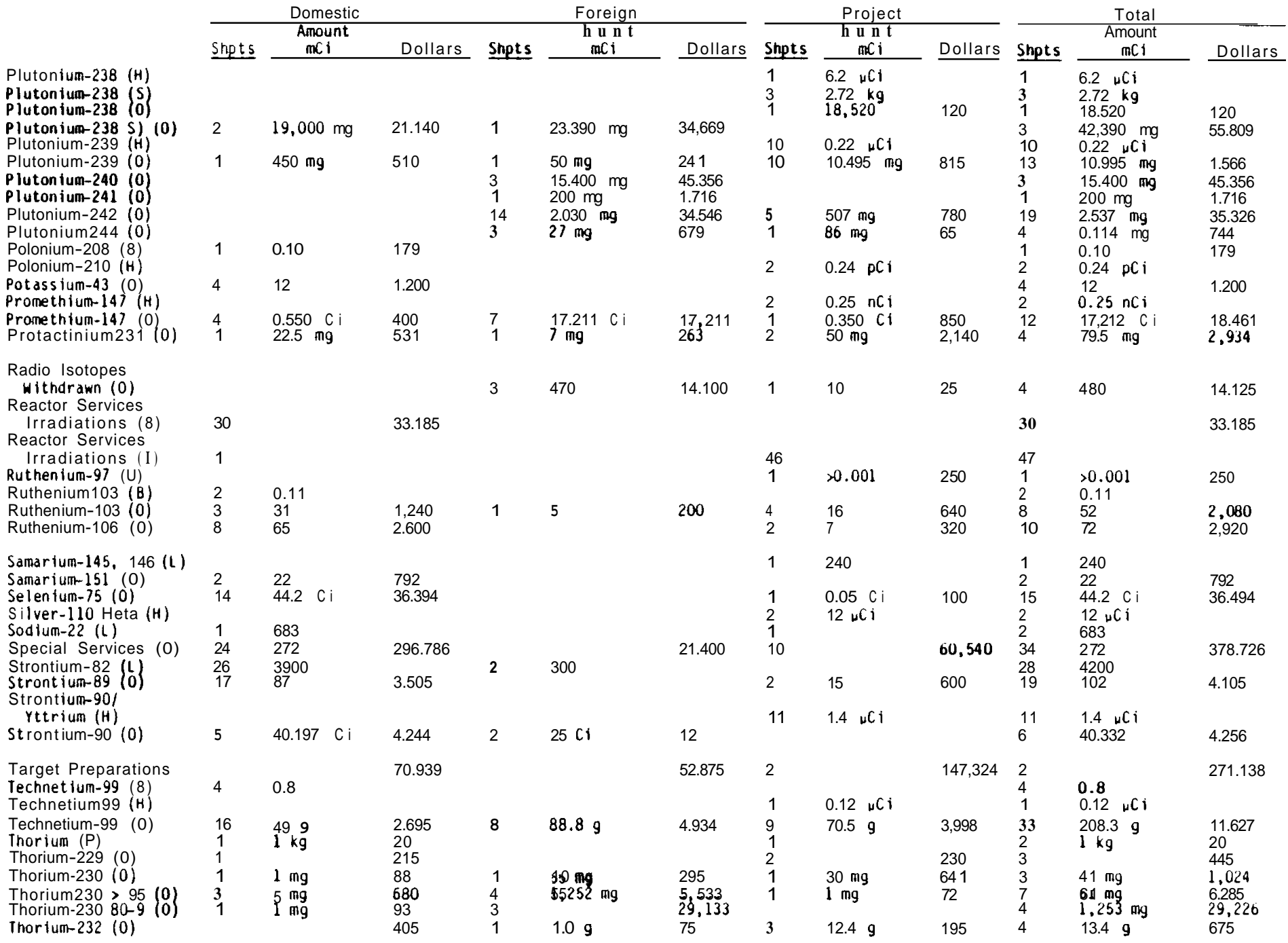


5. RADIOISOTOPE SALES AND TRANSFERS--FY1981

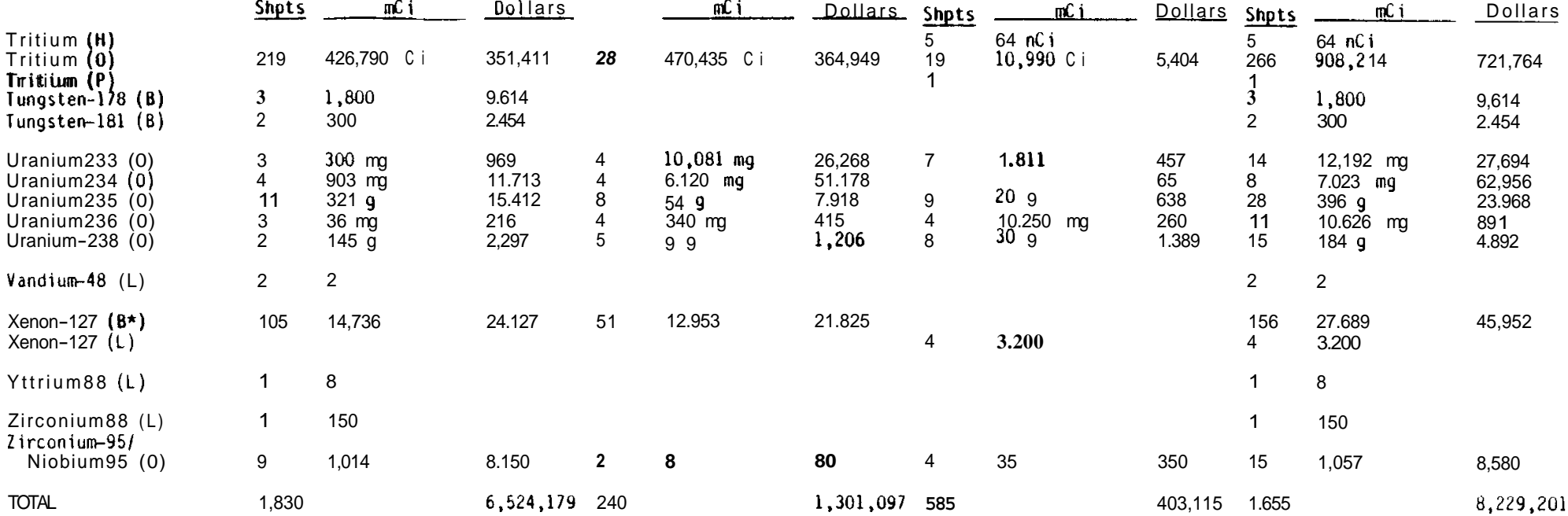

(a) 0ak Ridge billed.

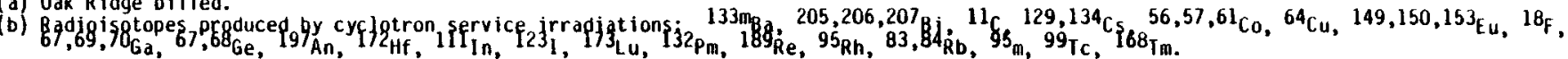

(c) Special services includes such items as target fabrication, source encapsulation, and preparation of special chemical forms. 
No. of

Copies

\section{OFFSITE}

H. Book

U.S. Nuclear Regulatory

Commission

Suite 202

Walnut Creek, CA 94596

\section{P. B. Brimhall}

DOE Idaho Operations Office

550 Second Street

Idaho Falls, ID 83401

G. Brown

U.S. Nuclear Regulatory

Commission

611 Ryan Plaza Drive

Suite 1000

Arlington, TX 76012

R. L. Clark

DOE Albuquerque Operations

Office

P.O. Box 5400

Albuquerque, M 87115

P. Colsmann

Brookhaven National Laboratory

Associated Universities, Inc.

Upton, NY 11973

DOE Office of the Assistant Secretary for International

Affairs (MS-7220C)

Washington, DC 20545

DOE Savannah River Operations Office

P.O. Box A

Aiken, SC 29801

27 DOE Technical Information Center

P.O. Box 62

Oak Ridge, TN 37830

B. Davis

U.S. Nuclear Regulatory

Commission

799 Roosevelt Road

Glen Ellyn, IL 60137
No. of

Copies

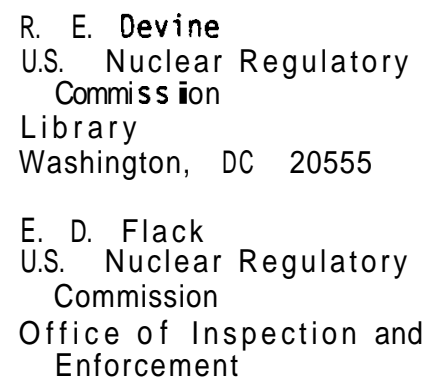

D. C. Graves

DOE Albuquerque Operations Office

P.O. Box 5400

Albuquerque, NM 87115

R. Grandfield DOE Dayton Area Office

P.O. Box 66

Miamisburg, $\mathrm{OH} 45342$

A. F. Grella

U.S. Department of Transportation

Washington, DC 20555

D. Hamilton Department of Health Education and Welfare

Food and Drug Administration

1901 Chapman Avenue

Mai 1stop HFX-70

Rockville, MD 20857 
No. of

Copies

R. A. Hasse

Idaho National Engineering Co.

EGE Idaho Inc.

P.O. Box 1625

Idaho Falls, ID 83401

J. C. Haugen

Argonne National Laboratory

9700 South Cass Avenue

Argonne, IL 60439

R. K. Heusser

DP-73

U.S. Department of Energy

Washington, DC 20545

D. R. Hopkins

U.S. Nuclear Regulatory

Commission

Washington, DC 20555

E. H. Kobisk

Oak Ridge National Laboratory

Union Carbide Corporation

P.O. Box X

Oak Ridge, TN 37830

R. F. Knott

DP-531

U.S. Department of Energy

Washington, DC 20585

E. E. Lamb

Oak Ridge National Laboratory

Union Carbide Corporation

P.O. Box X

Oak Ridge, $\mathbb{T N} 37830$

R. Lambrecht

Chemistry Department

Brookhaven National Laboratory

Upton, NY 11973

D. E. Large

DOE Oak Ridge Operations Office

P.O. Box E

Oak Ridge, TN 37830

C. Longmire

Los Alamos Scientic Laboratory

University of California

P.O. Box 1663

Los Alamos, NM 87545

K. W. MacMurdo

Savannah River Laboratory

- E. I. du Pont de Nemours Co.

Aiken, SC 29801
No. of

Copies

10 J. N. Maddox

Office of Energy Research ER-70 GTN

Office of Environment

U.S. Department of Energy

Washington, DC 20545

N. A. Matwiyoff

Los Alamos Scientific Laboratory

University of California

P.O. Box 1663

Los Alamos, NM 87544

J. W. Nehls

DOE Oak Ridge Operations Office

P.O. Box E

Oak Ridge, TN 37830

E Newman

Oak Ridge National Laboratory

Union Carbide Corporation

P.O. Box $X$

Oak Ridge, TN 37830

2 J. E. Ratledge

Oak Ridge National Laboratory

Union Carbide Corporation

P.O. Box X

Oak Ridge, TN 37830

J. E. Reid, (MA-843.3)

DOE Office of Controller

Washington, DC 20545

P. Richards

Brookhaven National Laboratory

Associated Universities, Inc.

Upton, NY 11973

B. Singer

U.S. Nuclear Regulatory

Commiss ion

Washington, OC 20555

G. Smith

U.S. Nuclear Regulatory

Commis s ion

631 Park Avenue

King of Prussia, PA 19406

L. G. Stang, Jr.

Brookhaven National Laboratory

Associated Universities, Inc.

Upton, NY 11973

J. Sutherland

U.S. Nuclear Regulatory

Commission

101 Marietta Street

Suite 3100

Atlanta, GA 30303 
No. of

Copies

A. N. Tse

U.S. Nuclear Regulatory Commi s si on

Washington, DC 20555

D. H. Turno

Savannah River Laboratory

E. I. du Pont de Nemours Co.

Aiken, SC 29801

R. E. Vallee

Mound Facility

Monsanto Research Corporation

P.0. Box 32

Miamisburg, OH 45342

E. Vejvoda

Rockwell International

Atomics International Division

Rocky Flats Plant

P.O. Box 464

Golden, CO 80401

E. พ. Yeats

Lawrence Berkeley Laboratory

University of California

Berkeley, CA 94720

ONSITE

2 DOE Richland Operations Office

ฟ. R. Pohlod

H. E. Ransom
No. of

Copies

Hanford Engineering Development Laboratory

D. Enge 1

UNC Nuclear Industries

P. A. Carlson

31 Pacific Northwest Laboratory

W. J. Bair

J. B. Brown

J. S. Burlison (15)

J. V. Filsinger

3. J. Fuquay

W. A. Glass

J. H. Jarrett

R. W. McKee

R. W. Perkins

E. J. Wheelwright

Technical Information (5)

Publishing Coordination js (2) 